\title{
WEAK SOLUTIONS TO MEAN CURVATURE FLOW RESPECTING OBSTACLES
}

\author{
MELANIE RUPFLIN AND OLIVER C. SCHNÜRER
}

\begin{abstract}
We consider the problem of evolving hypersurfaces by mean curvature flow in the presence of obstacles, that is domains which the flow is not allowed to enter. In this paper, we treat the case of complete graphs and explain how the approach of M. Sáez and the second author [19] yields a global weak solution to the original problem for general initial data and onesided obstacles.
\end{abstract}

\section{Contents}

1. Introduction

2. Definition of a solution

3. Main results and overview of the proof

4. Notations and geometry of submanifolds

5. Evolution equations

6. Estimates on the depth of penetration

7. $C^{1}$-estimates for the graphical flow: gradient fumction

8. Controlling the second fundamental form 15

9. Existence of approximate solutions 25

10. Proofs of the main results 27

11. Geometric interpretation: back to the original problem 28

References

\section{INTRODUCTION}

Given a hypersurface in Euclidean space we investigate how one can evolve this hypersurface by mean curvature flow if there are parts of space, so called obstacles, that the hypersurface is forbidden from entering.

To be more precise, let $\mathcal{P}$ be an open non-empty set in Euclidean space, not necessarily connected, nor bounded or regular and let $N_{0}$ be an initial hypersurface which is disjoint from $\mathcal{P}$. We then would like to evolve $N_{0}$ by a family of hypersurfaces $\left(N_{t}\right)_{t}$, locally described by parametrisations $F_{t}$, moving in normal direction, in such a way that

(1) $N_{t}$ satisfies (a weak form of) mean curvature flow

$$
\frac{d}{d t} F=-H \nu
$$

on the complement of the obstacle $\overline{\mathcal{P}}$.

(2) $N_{t}$ remains disjoint from the obstacle, $N_{t} \cap \mathcal{P}=\emptyset$.

Date: December 18, 2018.

2000 Mathematics Subject Classification. 53C44. 
(3) In points where the hypersurface touches the (closure of the) obstacle, the hypersurface evolves by mean curvature flow if this makes the hypersurfaces lift off the obstacle, but remains stationary otherwise, i. e. for $p \in \overline{\mathcal{P}} \cap N_{t}$ we would like to ask that

$$
\frac{d}{d t} F=\left\langle-H \nu, \nu_{\mathcal{P}}\right\rangle_{+} \cdot \nu_{\mathcal{P}}=(-H)_{+} \nu
$$

where $\nu_{\mathcal{P}}$ denotes the outwards pointing unit normal to $\partial \mathcal{P}$ (where defined) and $\langle a, b\rangle_{+}=\max (\langle a, b\rangle, 0)$.

A first approach to mean curvature flow with obstacles was carried out by L. Almeida, A. Chambolle, and M. Novaga [1] who constructed solutions based on a time-discretisation scheme for the corresponding partial differential inequality and obtained in particular short-time existence of $C^{1,1}$-solutions in certain settings. Furthermore, E. Spadaro [20] considered mean curvature flow with obstacles in order to investigate properties of mean convex sets. He used a time-discretisation to obtain a weak mean curvature flow of Caccioppoli sets and the focus of his work is on the properties of the limits as $t \rightarrow \infty$ of such weak solutions.

In the present paper we show that the ideas of M. Sáez and the second author [19] introduced for the study of standard mean curvature flow can be used to obtain a new approach for mean curvature flow with obstacles that avoids the study of singularities completely but allows us to show global existence of weak solutions for essentially all (reasonable) initial data and onesided obstacles.

The basic idea of the construction is the following: Given any initial ( $n$-dimensional) hypersurface $N_{0} \subset \mathbb{R}^{n+1}$ and an obstacle $\mathcal{P} \subset \mathbb{R}^{n+1}$ we lift the problem to one dimension higher by building complete graphs over both the obstacle and the region enclosed by the initial hypersurface $N_{0}$ which contains the obstacle, see Figure 1.

We then consider the new and simpler problem of flowing a graphical hypersurface $M_{0}$ in the presence of a graphical obstacle $\mathcal{O}$ for which we prove long-time existence of a viscosity solution. This solution of the graphical problem is obtained as a limit of flows that do not prohibit the penetration of the obstacle but only penalise it appropriately. A key part of the analysis of these approximate solutions carried out later on is to prove that they satisfy locally uniform spatial $C^{2}$-estimates. This implies in particular that the viscosity solution that we obtain is of class $C^{1,1}$ which, in view of the analysis of the corresponding stationary problem of C. Gerhardt [8], is optimal.

Similarly to [19], one can interpret the projection of this graphical flow $\left(M_{t}\right)_{t}$ in $\mathbb{R}^{n+2}$ to $\mathbb{R}^{n+1}$ as a weak solution $\left(N_{t}\right)_{t}$ for the original problem of evolving by mean curvature flow in $\mathbb{R}^{n+1}$ respecting the obstacle $\mathcal{P}$.

We note that the problem of evolving hypersurfaces by mean curvature in the presence of obstacles has been considered independently in the interesting papers [18] by G. Mercier and M. Novaga and [17] by G. Mercier that were first published at the same time as the present paper. The paper [18] also deals with the evolution of graphical hypersurfaces, though the employed techniques are very different from our approach and the focus of [18] is on the evolution of entire graphs that then form the basis for the construction of level set solutions in [17], rather then of complete graphs over time-dependent domains as needed in the present paper.

Since this paper was first published in September 2014 there have been further interesting developments related to the problem studied in the present paper: P. Logaritsch studied boundary value problems for graphical mean curvature flow with obstacles using time-discretisation in [15], while W. Maurer [16] generalised the methods of [19] to Dirichlet boundary value problems and graphs that are unbounded from both above and below. 


\section{Definition of a solution}

Definition 2.1 (Initial data). Given an open, possibly disconnected set $\mathcal{P} \subset \mathbb{R}^{n+1}$, we consider an initial hypersurface $N_{0} \subset \mathbb{R}^{n+1}$ which is disjoint from $\mathcal{P} \subset \mathbb{R}^{n+1}$ and an open, possibly unbounded and disconnected, set $\Omega_{0} \subset \mathbb{R}^{n+1}$, such that

$$
\partial \Omega_{0}=N_{0} \quad \text { and } \quad \mathcal{P} \subset \Omega_{0} .
$$

For the lifted problem in $\mathbb{R}^{n+2}$ we then consider initial data consisting of an obstacle $\mathcal{O}$ and an initial hypersurface $M_{0}$ with the following properties.

(i) The obstacle $\mathcal{O} \subset \mathbb{R}^{n+2}$ is given as

$$
\mathcal{O}=\left\{\left(\hat{x}, x^{n+2}\right) \in \mathbb{R}^{n+2}: x^{n+2}<\psi(\hat{x})\right\}
$$

for a function $\psi \in C_{l o c}^{1,1}(\mathcal{P})$ which is proper and bounded above.

In particular, $\psi(\hat{x}) \rightarrow-\infty$ for $\hat{x} \rightarrow \partial \mathcal{P}$ or $|\hat{x}| \rightarrow \infty$.

(ii) The initial hypersurface $M_{0} \subset \mathbb{R}^{n+2}$ is given as

$$
M_{0}=\text { graph } u_{0}
$$

for a locally Lipschitz function $u_{0}: \Omega_{0} \rightarrow \mathbb{R}$ which is proper, bounded above and fulfils

$$
u_{0} \geq \psi \quad \text { in } \quad \mathcal{P} \subset \Omega_{0}
$$

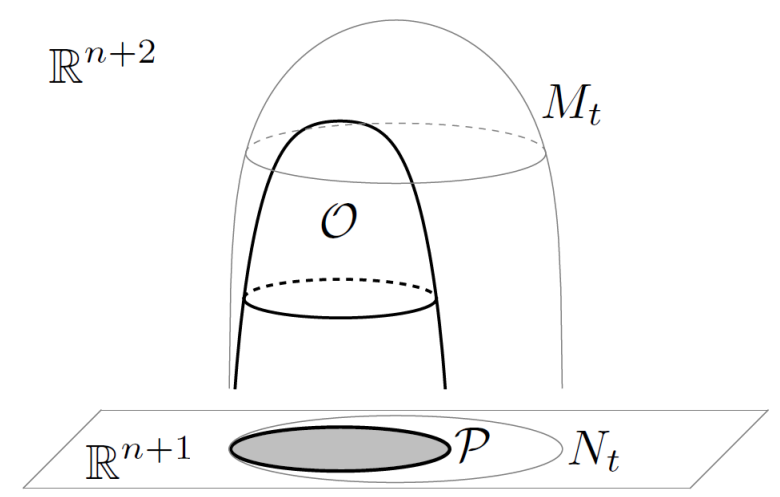

FiguRE 1. Graphical initial hypersurface $M_{0}$ and obstacle $\partial \mathcal{O}$ in $\mathbb{R}^{n+2}$ associated with the original data $N_{0}$ and $\mathcal{P}$.

We remark that there is no need to impose any regularity assumptions on either $\mathcal{P}$ or $\partial \Omega_{0}$ in order to obtain such lifted initial data $\mathcal{O}$ and $M_{0}$. Furthermore, $\mathcal{O}$ can and will be chosen so that $\partial \mathcal{O}$ has uniformly bounded second fundamental form if $\partial \mathcal{P}$ has uniformly bounded second fundamental form and a tubular neighbourhood with thickness uniformly bounded below. An analogous statement holds for $M_{0}$ and $N_{0}=\partial \Omega_{0}$.

We adapt the definition of a solution to graphical mean curvature flow from [19] to the situation with obstacles. We follow the convention that the obstacle lies below the solution, see e.g. [8], and therefore have to reflect the setting in [19]. In particular the evolving hypersurface $M_{t}=\left.\operatorname{graph} u(\cdot, t)\right|_{\Omega_{t}}$ will be represented by a pair $(\Omega, u)$, where $\Omega \subset \mathbb{R}^{n+1} \times[0, \infty)$ is a subset of space-time, $u(x, t)$ is defined for $(x, t) \in \Omega$ and $\Omega_{t} \subset \mathbb{R}^{n+1}$ is a time-slice of the space-time domain $\Omega$ as defined below. We refer to [19] for a more in depth discussion of the motivation behind the definition.

In the following definition we use standard notation: $H$ denotes the mean curvature of $M_{t}$ and $v=\left\langle\nu, e_{n+2}\right\rangle^{-1}$. For details we refer to Section 4 . 
Definition 2.2 (graphical mean curvature flow with obstacle).

(i) Domain of definition: Let $\Omega \subset \mathbb{R}^{n+1} \times[0, \infty)$ be a (relatively) open set. Set $\Omega_{t}:=\pi_{\mathbb{R}^{n+1}}\left(\Omega \cap\left(\mathbb{R}^{n+1} \times\{t\}\right)\right)$, where $\pi_{\mathbb{R}^{n+1}}: \mathbb{R}^{n+2} \rightarrow \mathbb{R}^{n+1}$ is the orthogonal projection to the first $n+1$ components. We require that $\mathcal{P} \subset \Omega_{t}$ for every $t \in[0, \infty)$.

(ii) The solution: A function $u: \Omega \rightarrow \mathbb{R}$ is called a solution to graphical mean curvature flow in $\Omega$ with initial value $u_{0}: \Omega_{0} \rightarrow \mathbb{R}$ and obstacle $(\mathcal{P}, \psi)$ or $\mathcal{O}$, if $u \in C_{l o c}^{0}(\Omega)$ satisfies

$$
\begin{cases}\min \left\{\dot{u}-\sqrt{1+|D u|^{2}} \cdot \operatorname{div}\left(\frac{D u}{\sqrt{1+|D u|^{2}}}\right), u-\psi\right\}=0 & \text { in } \Omega, \\ u(\cdot, 0)=u_{0} & \text { in } \Omega_{0},\end{cases}
$$

in the viscosity sense.

(iii) Maximality condition: A function $u: \Omega \rightarrow \mathbb{R}$ fulfils the maximality condition if $u \leq c$ for some $c \in \mathbb{R}$ and if $\left.u\right|_{\Omega \cap\left(\mathbb{R}^{n+1} \times[0, T]\right)}$ is proper for every $T>0$. An initial value $u_{0}: \Omega_{0} \rightarrow \mathbb{R}, \Omega_{0} \subset \mathbb{R}^{n+1}$, is said to fulfil the maximality condition if $w: \Omega_{0} \times[0, \infty) \rightarrow \mathbb{R}$ defined by $w(x, t):=u_{0}(x)$ fulfils the maximality condition.

(iv) Singularity resolving solution: $(\Omega, u)$, or equivalently $\left(M_{t}\right)_{t \geq 0}$ given by $M_{t}=\left.\operatorname{graph} u(\cdot, t)\right|_{\Omega_{t}} \subset \mathbb{R}^{n+2}$, is called a singularity resolving solution to mean curvature flow respecting the obstacle $\mathcal{O}$ if the conditions (i)-(iii) are fulfilled.

The formulation involving the minimum in (2.1) is a standard description for viscosity solutions to obstacle problems cf. [3, Example 1.7]. We remark that the above definition immediately implies that $u \geq \psi$ and that $\dot{u}+\sqrt{1+|D u|^{2}} \cdot H=0$ in the viscosity sense wherever $u>\psi$. Furthermore

Remark 2.3. For a $C^{2 ; 1}$-function $u$, the equation (2.1) is fulfilled if and only if $u$ is a solution to

$$
\begin{cases}\dot{u}=\sqrt{1+|D u|^{2}} \cdot \operatorname{div}\left(\frac{D u}{\sqrt{1+|D u|^{2}}}\right) \equiv-v \cdot H & \text { in } \Omega \backslash\left(\left(\Omega_{0} \times\{0\}\right) \cup \Gamma\right), \\ \dot{u}=v \cdot(-H)_{+} & \text {in }\left(\Omega \backslash\left(\Omega_{0} \times\{0\}\right)\right) \cap \Gamma, \\ u(\cdot, 0)=u_{0} \geq \psi & \text { in } \Omega_{0},\end{cases}
$$

where

$$
\Gamma:=\{(x, t) \in \Omega: u(x, t)=\psi(x)\}
$$

is the contact set between the evolving hypersurface and the obstacle.

In $C^{2 ; 1}$ and more generally for parabolic Hölder spaces, the first exponent refers to regularity in spatial and the second in time directions.

\section{Main Results AND OVERVIEW OF THE PROOF}

We prove

Theorem 3.1. Let $\mathcal{O}, \Omega_{0}$ and $u_{0}$ be an obstacle and an initial datum as in Definition 2.1. Then there exists a singularity resolving solution $(\Omega, u)$ with

$$
u \in C_{l o c}^{1,1 ; 0,1}\left(\Omega \backslash\left(\Omega_{0} \times\{0\}\right)\right) \cap C_{l o c}^{0}(\Omega)
$$

of mean curvature flow respecting the obstacle $\mathcal{O}$ for all times.

Furthermore, the evolving hypersurface $M_{t}:=\operatorname{graph} u(\cdot, t)$ is controlled in halfspaces of the form $\left\{x^{n+2}>\ell\right\}$ for arbitrary $\ell \in \mathbb{R}$ in the sense that $v=\left\langle\nu, e_{n+2}\right\rangle^{-1}$ and the second fundamental form $A$ of $M_{t}^{\ell}:=M_{t} \cap\left\{x^{n+2}>\ell\right\}$ satisfy

$$
\|v\|_{L^{\infty}\left(M_{t}^{\ell}\right)}+\sqrt{t} \cdot\|A\|_{L^{\infty}\left(M_{t}^{\ell}\right)} \leq C\left(u_{0}, \mathcal{O}, \ell\right) .
$$


Furthermore, if the initial hypersurface $M_{0}$ is $C_{l o c}^{1,1}$, then $M_{t}^{\ell}$ has uniformly controlled second fundamental form $\|A\|_{L^{\infty}\left(M_{t}^{\ell}\right)} \leq C\left(u_{0}, \mathcal{O}, \ell\right)$ up to time $t=0$.

In addition, for positive times, $u$ is smooth away from the contact set.

\section{Remark 3.2.}

(i) The regularity statement of Theorem 3.1 can be seen as the analogue of $C$. Gerhardt's $C^{1,1}$-regularity result [8] for solutions of the stationary obstacle problem. The simple example of a rope spanned over a circle illustrates in both cases that the spatial $C^{1,1}$-regularity is optimal.

(ii) As $C^{1,1}$-functions are twice differentiable almost everywhere, the second fundamental form is defined almost everywhere and the above $L^{\infty}$-bounds on the second fundamental form and the gradient are equivalent to local $C^{1,1}$-bounds.

As it is of interest to consider not only complete but also entire graphs, we prove additionally

Theorem 3.3. Let $u_{0}: \mathbb{R}^{n+1} \rightarrow \mathbb{R}$ be bounded and Lipschitz continuous. Assume that $u_{0}$ is constant outside a compact subset of $\mathbb{R}^{n+1}$. Let $\psi: \mathbb{R}^{n+1} \rightarrow \mathbb{R}$ be a function describing an obstacle as in Definition 2.1. Assume furthermore that $u_{0} \geq$ $\psi$. Then there exists a uniformly continuous viscosity solution $u: \mathbb{R}^{n+1} \times[0, \infty) \rightarrow \mathbb{R}$ of mean curvature flow with obstacle

$$
\min \left\{\dot{u}-\sqrt{1+|D u|^{2}} \cdot \operatorname{div}\left(\frac{D u}{\sqrt{1+|D u|^{2}}}\right), u-\psi\right\}=0
$$

with $u(\cdot, 0)=u_{0}$. Furthermore

$$
\|u\|_{L^{\infty}\left(M_{t}\right)}+\|v\|_{L^{\infty}\left(M_{t}\right)}+\sqrt{t} \cdot\|A\|_{L^{\infty}\left(M_{t}\right)} \leq C\left(u_{0}, \psi\right) .
$$

Theorem 3.3 could be used to construct viscosity solutions for mean curvature flow with obstacles based on the level set approach. Such solutions were recently constructed in [17].

Of course, in the absence of an obstacle, this result is a special case of [5].

The approach we use to construct a solution of mean curvature flow with obstacles in the graphical setting is by penalisation. We obtain the desired viscosity solution as a limit of solutions to problems which allow a penetration of the obstacle, but penalise it by stronger and stronger normal vector fields trying to push the hypersurface back out of the obstacle.

More precisely, we fix a function $\beta \in C^{\infty}(\mathbb{R},[0, \infty))$, supported in $(-\infty, 0]$ with $\beta^{\prime \prime}$ non-increasing, and thus in particular satisfying $\beta^{\prime \prime}>0$ whenever $\beta>0$, and consequently also $\beta^{\prime}<0$.

We furthermore define dist $_{\partial \mathcal{O}}$ to be the signed distance function to the boundary of $\mathcal{O}$ chosen so that dist $\partial \mathcal{O}$ is negative in $\mathcal{O}$.

Given $\varepsilon>0$ we then consider the flow

$$
\frac{d}{d t} F=-\left(H-\alpha_{\varepsilon}\right) \cdot \nu=\Delta F+\alpha_{\varepsilon} \nu
$$

where

$$
\alpha_{\varepsilon}(p):=\beta_{\varepsilon}\left(\operatorname{dist}_{\partial \mathcal{O}}(p)\right), \quad \beta_{\varepsilon}(s)=\beta\left(\frac{s}{\varepsilon}\right)
$$

and where $\Delta$ is the Laplacian on the evolving submanifold so that $-H \nu=\Delta F$.

We stress that our penalisation depends on the Euclidean distance to $\partial \mathcal{O} \subset \mathbb{R}^{n+2}$ and not on the graphical one, i. e. not on $u(x, t)-\psi(x)$. This feature of the construction is crucial in order to be able to deal with complete graphs over possibly bounded domains.

While solutions to the penalised flow can sink into the obstacle, we shall show in Section 6 that the depth of this penetration is of order $O(\varepsilon)$. In Section 7 we shall 
then prove that the gradient function of these approximate solutions is bounded uniformly in time and locally in space. Similar $C^{1,1}$-estimates will be deduced in the following Section 8. We stress that these estimates are independent of the parameter $\varepsilon$ of the penalisation which thus immediately gives $C^{1,1}$ regularity also for our viscosity solution of mean curvature flow with obstacles which we obtain in the limit $\varepsilon \searrow 0$, see Section 10 .

While we will state and prove these results only for smooth obstacles, all the estimates derived in Sections 7 and 8 depend only on the local $C^{2}$-norm of $\psi$, so we are able to reduce the proof of Theorems 3.1 and 3.3 to the case of smooth obstacles and an approximation argument carried out later on in Section 10. In particular we will assume from now on that $\psi$ is smooth unless stated otherwise.

\section{Notations AND GEOMETRY OF SUBMANifolds}

We use $F=F(x, t)=\left(F^{\alpha}\right)_{1 \leq \alpha \leq n+2}$ to denote the time-dependent embedding vector of a manifold $M^{n+1}$ into $\mathbb{R}^{n+2}$ and $\frac{d}{d t} F=\dot{F}$ for its total time derivative. We set $M_{t}:=F(M, t) \subset \mathbb{R}^{n+2}$ and will often identify an embedded manifold with its image. We will assume that $F$ is smooth. We assume furthermore that $M^{n+1}$ is smooth and orientable. The embedding $F(\cdot, t)$ induces a metric $g=\left(g_{i j}\right)_{1 \leq i, j \leq n+1}$ on $M_{t}$. We denote by $\nabla$ the Levi-Civitá connection on $\left(M_{t}, g(t)\right)_{t}$ and the induced bundles while we write $\bar{\nabla}$ for the gradient on the ambient space $\mathbb{R}^{n+2}$.

We choose $\nu=\left(\nu^{\alpha}\right)_{1 \leq \alpha \leq n+2}$ to be the upward pointing unit normal vector to $M_{t}$ at $x \in M_{t}$.

The second fundamental form $A$ is then characterized through the Gauß equation

$$
\nabla_{i} \nabla_{j} F=-A_{i j} \nu
$$

or, equivalently, the Weingarten equation

$$
\nabla_{i} \nu=A_{i l} g^{l k} \nabla_{k} F=A_{i}^{k} \nabla_{k} F
$$

Here and in the following, we raise and lower indices using the metric and its inverse $\left(g^{i j}\right)$ and utilize the Einstein summation convention to sum over repeated upper and lower indices.

Throughout the paper, Latin indices range from 1 to $n+1$ and refer to geometric quantities on the hypersurface, while Greek indices refer to the components in fixed Euclidean coordinates in the ambient space $\mathbb{R}^{n+2}$.

We define the mean curvature $H$ by $H=g^{i j} A_{i j}$ and compute the norm of the second fundamental form through $|A|^{2}=A_{i j} g^{j k} A_{k l} g^{i l}$.

Finally, given a function $f$ defined on the ambient space $\mathbb{R}^{n+2}$ we write $\nabla f$ for the derivative of $\left.f\right|_{M_{t}}$ on $M_{t}$ which can equivalently be computed as the projection

$$
\nabla f=P_{T M}(\bar{\nabla} f)=\bar{\nabla} f-\langle\bar{\nabla} f, \nu\rangle \nu,
$$

of the ambient gradient to the tangent space of the evolving hypersurface $M_{t}$. Here we use in the last equality that this orthogonal projection $P_{T M}: \mathbb{R}^{m+2} \rightarrow T_{p} M_{t}$, $p \in M$, can be expressed in terms of the normal as $P_{T M}(X)=X-\langle X, \nu\rangle \nu$, where $\langle\cdot, \cdot\rangle$ denotes the Euclidean inner product on $\mathbb{R}^{n+2}$. Furthermore we will consider the gradient $\nabla f(p, t)$ of functions $f$, be they defined on all of $\mathbb{R}^{n+2}$ or only on $M_{t}$, as a vector in either $T_{p} M_{t}$ or in $\mathbb{R}^{n+2}$ as convenient and without changing the notation. Similarly, we will evaluate geometric quantities either at $(x, t) \in M \times[0, \infty)$ or at $p=F(x, t) \in M_{t} \subset \mathbb{R}^{n+2}$.

As the topology of our solutions may change, we only require that solutions to (3.2) are parametrised over a base manifold $M$ locally in space and time. 
We shall also use that the Gauß equation allows us to express the Riemannian curvature tensor of the hypersurface in terms of the second fundamental form

$$
R_{i j k l}=A_{i k} A_{j l}-A_{i l} A_{j k} .
$$

Throughout the paper, expressions like $\nabla_{i} \nabla_{j} A_{k l}$ are to be understood as first computing the covariant derivatives of the tensor $A$ and then evaluating it in the indicated directions of the standard basis vector fields.

\section{Evolution EQUATIONS}

In this section we collect the evolution equations of the various geometric quantities such as gradient function, second fundamental form, etc. As the corresponding formulas for mean curvature flow, and more generally for graphical flows moving in normal direction, are well known, see $[4,9,11]$, we will mainly analyse the influence of the penalisation $\alpha_{\varepsilon}$.

We remark that the distance function $\operatorname{dist}_{\partial \mathcal{O}}$ as well as its level sets are $C_{\text {loc }}^{2}$ in a neighbourhood of $\partial \mathcal{O}$ and that throughout this section we shall only consider points which, if they are in $\mathcal{O}$, are contained in such a neighbourhood. We will later justify this assumption as a consequence of Lemma 6.1.

To begin with, we define the height function of the evolving hypersurface by

$$
U:=\left\langle F, e_{n+2}\right\rangle .
$$

For graphical hypersurfaces, the penalised flow (3.2) can be rewritten in terms of $U$ as

$$
\frac{d}{d t} U-\Delta U=\alpha_{\varepsilon}\left\langle\nu, e_{n+2}\right\rangle=\frac{\alpha_{\varepsilon}}{v},
$$

$v$ the gradient function introduced above.

For a family of hypersurfaces moving with normal velocity $f$,

$$
\frac{d}{d t} F=-f \cdot \nu
$$

$f$ any function defined on the evolving hypersurfaces, it is well-known that the metric evolves by $\frac{d}{d t} g_{i j}=-2 f A_{i j}$ which becomes

$$
\frac{d}{d t} g_{i j}=-2\left(H-\alpha_{\varepsilon}\right) A_{i j}
$$

in our case. The normal evolves by

$$
\frac{d}{d t} \nu=\nabla f
$$

so using the identity

$$
\Delta \nu=-|A|^{2} \nu+\nabla H,
$$

valid for arbitrary hypersurfaces in Euclidean space, we obtain in this more general context of (5.1) that

$$
\left(\frac{d}{d t}-\Delta\right) \nu=|A|^{2} \nu+\nabla(f-H),
$$

which for our flow translates to

Lemma 5.1. For hypersurfaces evolving according to (3.2), $\nu$ fulfills

$$
\frac{d}{d t} \nu-\Delta \nu=|A|^{2} \nu-\nabla \alpha_{\varepsilon}
$$

or, equivalently, written out in local coordinates

$$
\frac{d}{d t} \nu^{\beta}-\Delta \nu^{\beta}=|A|^{2} \nu^{\beta}-\bar{\nabla}_{\gamma} \alpha_{\varepsilon} \nabla_{i} F^{\gamma} g^{i j} \nabla_{j} F^{\beta} .
$$


With $\alpha_{\varepsilon}$ given by $\alpha_{\varepsilon}=\beta_{\varepsilon} \circ$ dist $_{\partial \mathcal{O}}$, its derivative in a point $p \in M_{t} \cap \mathcal{O}$ is determined in terms of $\nu_{\mathcal{O}}=\bar{\nabla}$ dist $_{\mathcal{O}}$ (where defined) which describes the outwards unit normal to the level set

$$
\partial \mathcal{O}_{\delta}:=\left\{y \in \mathbb{R}^{n+2}: \operatorname{dist}_{\partial \mathcal{O}}(y)=-\delta\right\}
$$

which contains $p$. Namely,

$$
\nabla \alpha_{\varepsilon}=\beta_{\varepsilon}^{\prime} \cdot \nabla \operatorname{dist}_{\partial \mathcal{O}}=\beta_{\varepsilon}^{\prime} \cdot P_{T M}\left(\nu_{\mathcal{O}}\right)=\beta_{\varepsilon}^{\prime} \cdot\left(\nu_{\mathcal{O}}-\left\langle\nu_{\mathcal{O}}, \nu\right\rangle \nu\right),
$$

or equivalently, working in local coordinates, $\nabla_{j} \alpha_{\varepsilon}=\beta_{\varepsilon}^{\prime} \cdot\left\langle\nu_{\mathcal{O}}, \nabla_{j} F\right\rangle$.

Outside of $\mathcal{O}$, the derivative of $\alpha_{\varepsilon}$ vanishes.

For graphical solutions of (3.2), or more generally of (5.1), we then consider the 'gradient function' $v$ defined by $v=\left\langle\nu, e_{n+2}\right\rangle^{-1}$ which, by (5.3), satisfies

$$
\begin{aligned}
\left(\frac{d}{d t}-\Delta\right) v & =-\left\langle\nu, e_{n+2}\right\rangle^{-2} \cdot\left\langle\left(\frac{d}{d t}-\Delta\right) \nu, e_{n+2}\right\rangle-2\left\langle\nu, e_{n+2}\right\rangle^{-3}\left|\nabla\left\langle\nu, e_{n+2}\right\rangle\right|^{2} \\
& =-v^{2} \cdot\left[\left\langle\nu, e_{n+2}\right\rangle|A|^{2}+\left\langle\nabla(f-H), e_{n+2}\right\rangle\right]-2 v^{3}\left|\nabla\left(v^{-1}\right)\right|^{2} \\
& =-|A|^{2} \cdot v-2 \frac{|\nabla v|^{2}}{v}-\left\langle\nabla(f-H), e_{n+2}\right\rangle \cdot v^{2} .
\end{aligned}
$$

We shall later use that we can express $\nabla v$ in terms of the second fundamental form as

$$
\nabla_{i} v=-v^{2}\left\langle\nabla_{i} \nu, e_{n+2}\right\rangle=-v^{2} A_{i}^{k}\left\langle\nabla_{k} F, e_{n+2}\right\rangle=-v^{2} A_{i}^{k} \nabla_{k} U
$$

but for now only need the conclusion that

Lemma 5.2. For graphical hypersurfaces evolving according to (3.2), the gradient function $v=\left\langle\nu, e_{n+2}\right\rangle^{-1}$ fulfills

$$
\frac{d}{d t} v-\Delta v=-|A|^{2} v-\frac{2}{v}|\nabla v|^{2}+v^{2}\left\langle\nabla \alpha_{\varepsilon}, e_{n+2}\right\rangle .
$$

Compared with standard mean curvature flow we thus obtain an additional term that contains a derivative of the penalty function and which may thus become arbitrarily large in the limit $\varepsilon \searrow 0$.

However, as illustrated in Figure 2, in a point where the evolving hypersurface is 'steeper' than the obstacle, the penalisation helps to reduce $v$, because $\alpha_{\varepsilon}$ grows with increasing (negative) distance to $\partial \mathcal{O}$.

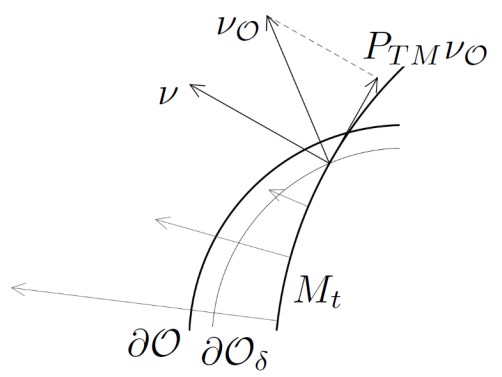

Figure 2. Penalising vectorfield and normals in a point where $v \geq v_{\nu}$.

More precisely, we obtain

Remark 5.3. Given a point $p \in \partial \mathcal{O}_{\delta}$ in a neighbourhood of which $\partial \mathcal{O}_{\delta}$ is a $C^{1}$ graph we let $v_{\mathcal{O}}:=\left\langle\nu_{\mathcal{O}}, e_{n+2}\right\rangle^{-1}$ be the gradient function (of the level sets) of the obstacle. Then at each point $p \in \mathcal{O} \cap M_{t}$ where

$$
v \geq v_{\mathcal{O}}
$$


we have

$$
\left\langle\nabla \alpha_{\varepsilon}, e_{n+2}\right\rangle \leq 0
$$

Proof. Since both the evolving hypersurface and the level sets of the obstacle are graphical and thus $v, v_{\mathcal{O}}$ are well defined and positive we can use (5.4) to compute

$$
\left\langle\nabla \alpha_{\varepsilon}, e_{n+2}\right\rangle=\beta_{\varepsilon}^{\prime}\left(\left\langle\nu_{\mathcal{O}}, e_{n+2}\right\rangle-\left\langle\nu_{\mathcal{O}}, \nu\right\rangle \cdot\left\langle\nu, e_{n+2}\right\rangle\right)=\beta_{\varepsilon}^{\prime} \cdot\left(\frac{1}{v_{\mathcal{O}}}-\frac{\left\langle\nu_{\mathcal{O}}, \nu\right\rangle}{v}\right)
$$

which gives the claim as $\beta_{\varepsilon}^{\prime} \leq 0$.

We finally turn to the evolution equation satisfied by the norm of the second fundamental form.

It is well-known that $|A|^{2}$ evolves along a normal flow (5.1) according to

$$
\frac{d}{d t}|A|^{2}=2 f A_{i}^{k} A_{j}^{i} A_{k}^{j}+2 A^{i j} \nabla_{i} \nabla_{j} f
$$

as well as that

$$
\Delta|A|^{2}=2 A^{i j} \nabla_{i} \nabla_{j} H+2|\nabla A|^{2}+2 H A_{i}^{k} A_{j}^{i} A_{k}^{j}-2|A|^{4} .
$$

This implies the general formula

$$
\left(\frac{d}{d t}-\Delta\right)|A|^{2}=-2 A^{i j} \nabla_{i} \nabla_{j}(H-f)-2(H-f) A_{i}^{k} A_{j}^{i} A_{k}^{j}+2|A|^{4}-2|\nabla A|^{2}
$$

which in our case becomes

Lemma 5.4. For hypersurfaces evolving by the penalised flow (3.2), the norm of the second fundamental form fulfils

$$
\left(\frac{d}{d t}-\Delta\right)|A|^{2}=-2|\nabla A|^{2}+2|A|^{4}-2 \alpha_{\varepsilon} A_{i}^{k} A_{j}^{i} A_{k}^{j}-2 \nabla_{i} \nabla_{j} \alpha_{\varepsilon} A^{i j} .
$$

The last term in this equation, given as the covariant derivative of the vector field $\nabla \alpha_{\varepsilon} \in \Gamma(T M)$, needs to be analysed carefully as it contains a second order derivative of the penalty function. As such it can be of order $\varepsilon^{-2}$ at points in the obstacle which might be reached by the evolving hypersurface, compare also Section 6.

The second covariant derivative of the penalisation function $\alpha_{\varepsilon}$ is given by $(5.8)$

$$
\begin{aligned}
\nabla_{i} \nabla_{j} \alpha_{\varepsilon} & =\nabla_{i}\left(\bar{\nabla}_{\nabla_{j} F} \alpha_{\varepsilon}\right)=\nabla_{i}\left(\left(\beta_{\varepsilon}^{\prime} \circ \operatorname{dist}_{\partial \mathcal{O}}\right) \cdot\left\langle\nu_{\mathcal{O}}, \nabla_{j} F\right\rangle\right) \\
& =\beta_{\varepsilon}^{\prime \prime} \cdot\left\langle\nu_{\mathcal{O}}, \nabla_{i} F\right\rangle \cdot\left\langle\nu_{\mathcal{O}}, \nabla_{j} F\right\rangle+\beta_{\varepsilon}^{\prime} \cdot\left\langle\bar{\nabla}_{\nabla_{i} F} \nu_{\mathcal{O}}, \nabla_{j} F\right\rangle+\beta_{\varepsilon}^{\prime}\left\langle\nu_{\mathcal{O}}, \nabla_{i} \nabla_{j} F\right\rangle .
\end{aligned}
$$

The last term in this formula is given by

$$
\beta_{\varepsilon}^{\prime}\left\langle\nu_{\mathcal{O}}, \nabla_{i} \nabla_{j} F\right\rangle=-\beta_{\varepsilon}^{\prime} A_{i j}\left\langle\nu_{\mathcal{O}}, \nu\right\rangle .
$$

For a better understanding of the penultimate term in (5.8), we choose an orthonormal basis $\left(e_{a}\right)$ of the tangent space to the level set $\partial \mathcal{O}_{\delta}$ which contains our point $p$ and write

$$
\nabla_{i} F=\left\langle\nabla_{i} F, e_{a}\right\rangle \delta^{a b} e_{b}+\left\langle\nabla_{i} F, \nu_{\mathcal{O}}\right\rangle \cdot \nu_{\mathcal{O}}
$$

In the resulting formula

$$
\bar{\nabla}_{\nabla_{i} F} \nu_{\mathcal{O}}=\left\langle\nabla_{i} F, e_{a}\right\rangle \delta^{a b} \cdot \bar{\nabla}_{e_{b}} \nu_{\mathcal{O}}+\left\langle\nabla_{i} F, \nu_{\mathcal{O}}\right\rangle \bar{\nabla}_{\nu_{\mathcal{O}}} \nu_{\mathcal{O}}
$$

the first term contains

$$
\bar{\nabla}_{e_{b}} \nu_{\mathcal{O}}=\left\langle\bar{\nabla}_{e_{b}} \nu_{\mathcal{O}}, e_{c}\right\rangle \delta^{c d} e_{d}=A_{b c}^{\mathcal{O}} \delta^{c d} e_{d},
$$


the (locally) bounded second fundamental form of the obstacle (or rather its level set $\partial \mathcal{O}_{\delta}$ ), while the second term can be seen to vanish identically; indeed since $\mid \bar{\nabla}$ dist $\left._{\partial \mathcal{O}}\right|^{2} \equiv 1$ we obtain for every $\gamma=1, \ldots, n+2$

$$
\left(\bar{\nabla}_{\nu_{\mathcal{O}}} \nu_{\mathcal{O}}\right)^{\gamma}=\nu_{\mathcal{O}}^{\eta} \frac{\partial}{\partial y^{\eta}} \nu_{\mathcal{O}}^{\gamma}=\sum_{\eta=1}^{n+2}\left(\frac{\partial}{\partial y^{\eta}} \frac{\partial}{\partial y^{\gamma}} \operatorname{dist}_{\partial \mathcal{O}}\right) \frac{\partial}{\partial y^{\eta}} \operatorname{dist}_{\partial \mathcal{O}}=\frac{1}{2} \frac{\partial}{\partial y^{\gamma}}\left|\bar{\nabla} \operatorname{dist}_{\partial \mathcal{O}}\right|^{2}=0 .
$$

Thus we can express the coefficient in the penultimate term in (5.8)

$$
\left\langle\bar{\nabla}_{\nabla_{i} F} \nu_{\mathcal{O}}, \nabla_{j} F\right\rangle=A_{b c}^{\mathcal{O}} \delta^{c d} \delta^{a b}\left\langle e_{a}, \nabla_{i} F\right\rangle \cdot\left\langle e_{d}, \nabla_{j} F\right\rangle=: \tilde{A}_{i j}^{\mathcal{O}},
$$

$i, j \in\{1, \ldots, n+1\}$, in terms of a tensor $\tilde{A}^{\mathcal{O}}$ which is controlled by $A^{\mathcal{O}}$.

All in all, the derivative of the penalisation is thus given by

$$
\nabla_{i} \nabla_{j} \alpha_{\varepsilon}=\beta_{\varepsilon}^{\prime \prime}\left\langle\nu_{\mathcal{O}}, \nabla_{i} F\right\rangle \cdot\left\langle\nu_{\mathcal{O}}, \nabla_{j} F\right\rangle+\beta_{\varepsilon}^{\prime} \tilde{A}_{i j}^{\mathcal{O}}-\beta_{\varepsilon}^{\prime} A_{i j}\left\langle\nu_{\mathcal{O}}, \nu\right\rangle
$$

which, once inserted into (5.7), results in

Lemma 5.5. For hypersurfaces evolving by the penalised flow (3.2), we have

$$
\begin{aligned}
\left(\frac{d}{d t}-\Delta\right)|A|^{2}= & -2|\nabla A|^{2}+2|A|^{4}-2 \alpha_{\varepsilon} A_{i}^{k} A_{j}^{i} A_{k}^{j} \\
(5.11) & -2 \beta_{\varepsilon}^{\prime \prime}\left\langle\nu_{\mathcal{O}}, \nabla_{i} F\right\rangle \cdot\left\langle\nu_{\mathcal{O}}, \nabla_{j} F\right\rangle A^{i j}-2 \beta_{\varepsilon}^{\prime} \tilde{A}_{i j}^{\mathcal{O}} A^{i j}+2 \beta_{\varepsilon}^{\prime}\left\langle\nu_{\nu} \nu_{\mathcal{O}}\right\rangle|A|^{2},
\end{aligned}
$$

where $\tilde{A}_{i j}^{\mathcal{O}}$ is given by $(5.9)$.

Contrary to the evolution equation for the gradient function, we cannot expect the additional terms to have a sign, so deriving suitable a priori bounds on the second fundamental form will be one of the main tasks in the analysis of the penalised flow (3.2). As we shall see, we can deal with this problem by considering a modified second fundamental form quantity which depends also on the penalty function itself.

For this we shall in particular need the evolution equation of the penalty function itself which is given by

Lemma 5.6. For hypersurfaces evolving by the penalised flow (3.2), we have

$$
\left(\frac{d}{d t}-\Delta_{M}\right) \alpha_{\varepsilon}=\beta_{\varepsilon}^{\prime} \alpha_{\varepsilon}\left\langle\nu_{\mathcal{O}}, \nu\right\rangle-\beta_{\varepsilon}^{\prime \prime}\left|P_{T M} \nu_{\mathcal{O}}\right|^{2}-\beta_{\varepsilon}^{\prime} \tilde{A}_{i j}^{\mathcal{O}} g^{i j} .
$$

Observe that the second term of this evolution equation gives a strong negative contribution (scaling as $\varepsilon^{-2}$ ) in points of the obstacle where the evolving hypersurface is not tangential to the level sets of the obstacle.

Proof. The formulas for the derivatives of the penalty function, see (5.10) and the formula following (5.4), immediately imply that

$$
\begin{aligned}
\left(\frac{d}{d t}-\Delta_{M}\right) \alpha_{\varepsilon} & =\beta_{\varepsilon}^{\prime}\left\langle\nu_{\mathcal{O}},\left(\frac{d}{d t}-\Delta_{M}\right) F\right\rangle-\beta_{\varepsilon}^{\prime \prime} \cdot\left\langle\nu_{\mathcal{O}}, \nabla_{i} F\right\rangle \cdot\left\langle\nu_{\mathcal{O}}, \nabla_{j} F\right\rangle g^{i j}-\beta_{\varepsilon}^{\prime} \cdot \tilde{A}_{i j}^{\mathcal{O}} g^{i j} \\
& =\beta_{\varepsilon}^{\prime} \alpha_{\varepsilon}\left\langle\nu_{\mathcal{O}}, \nu\right\rangle-\beta_{\varepsilon}^{\prime \prime}\left|P_{T M} \nu_{\mathcal{O}}\right|^{2}-\beta_{\varepsilon}^{\prime} \tilde{A}_{i j}^{\mathcal{O}} g^{i j}
\end{aligned}
$$

as claimed.

\section{Estimates on the DePth OF PENETRATion}

We shall later obtain the desired viscosity solution as limit of solutions to Dirichlet problems for (3.2) to be solved on larger and larger balls $B_{R}(0)$ where we will truncate the initial map $u_{0}$ at levels $L \ll 0$. In this context we shall always assume that $R$ is sufficiently large so that $\psi<L$ outside $B_{R}(0)$.

We prove the following bound for the amount that the evolving hypersurface can sink into the obstacle. 
Lemma 6.1. For any height $\ell \in \mathbb{R}$, there exists a number $C_{0}(\ell) \in(1, \infty)$ with the following property:

For any $L \in(-\infty, \ell)$ and $R>0$ as above, there exists $\varepsilon_{0}(L)>0$, such that for $0<\varepsilon \leq \varepsilon_{0}(L)$ any hypersurface $M_{t}=\operatorname{graph}\left(u_{\varepsilon, R}^{L}(\cdot, t)\right)$ evolving according to

$$
\begin{cases}\dot{u}=\sqrt{1+|D u|^{2}} \cdot\left(\operatorname{div}\left(\frac{D u}{\sqrt{1+|D u|^{2}}}\right)+\alpha_{\varepsilon}\right) & \text { in } B_{R}(0) \times[0, T), \\ u=L & \text { on } \partial B_{R}(0) \times[0, T), \\ u(\cdot, 0)=u_{0} \geq \max \{\psi, L\} & \text { in } B_{R}(0),\end{cases}
$$

satisfies

$$
\operatorname{dist}_{\partial \mathcal{O}}(p) \geq-C_{0}(\ell) \cdot \varepsilon
$$

in any point $p \in M_{t} \cap\left\{x^{n+2} \geq \ell\right\}$ and for all times $t \in[0, T)$.

We stress that the level $L$ at which we truncate the hypersurface only determines the range of admissible parameters $\varepsilon$, but that the bounds on the depth of penetration on $\left\{x^{n+2}>\ell\right\}$ are independent of $L$. To achieve this, we shall compare the evolving hypersurface with deformed level sets to $\partial \mathcal{O}$ of the following type.

Lemma 6.2. Given any function $f_{0} \in C_{\text {loc }}^{2}\left(\mathbb{R}^{n+2}, \mathbb{R}_{+}\right)$and any number $\varepsilon>0$, we let

$$
\mathcal{S}_{\varepsilon}:=\left\{p \in \mathbb{R}^{n+2}: \operatorname{dist}_{\partial \mathcal{O}}(p)=-\varepsilon f_{0}(p)\right\} .
$$

Then for any $L>-\infty$ and $\delta>0$, there exists a number $\varepsilon_{1}=\varepsilon_{1}\left(L, f_{0}, \delta\right)>0$ such that for any $|\varepsilon|<\varepsilon_{1}$ the hypersurfaces

$$
S_{\varepsilon} \cap\left\{x^{n+2}>L\right\}
$$

are of class $C^{2}$ with second fundamental form bounded by

$$
\left|A^{\mathcal{S}_{\varepsilon}}\right|(p) \leq(1+\delta) \cdot\left|A^{\mathcal{O}}\right|(p)+\delta
$$

for any $p \in\left\{x^{n+2}>L\right\} \cap \mathcal{S}_{\varepsilon}$, where $A^{\mathcal{O}}$ denotes the second fundamental form of the level set of $\operatorname{dist}_{\partial \mathcal{O}}$ that contains $p$.

In particular, there is a number $\varepsilon_{2}>0$ depending only on $L$, the function $f_{0}$ and on $\sup _{\partial \mathcal{O} \cap\left\{x^{n+2}>L-1\right\}}\left|A^{\mathcal{O}}\right|$ so that

$$
\left|A^{\mathcal{S}_{\varepsilon}}\right|(p) \leq 2 \cdot\left(\sup _{B_{1}(p) \cap \partial \mathcal{O}}\left|A^{\mathcal{O}}\right|+1\right)
$$

for $p \in \mathcal{S}_{\varepsilon} \cap\left\{x^{n+2}>L\right\}$ and $|\varepsilon|<\varepsilon_{2}$.

Proof. We first recall that given any function $w \in C^{2}\left(\mathbb{R}^{n+2}\right)$ and a point $p_{0} \in \mathbb{R}^{n+2}$ such that $D w\left(p_{0}\right) \neq 0$ one can compute the second fundamental form of the (locally $C^{2}$-) hypersurface

by

$$
\left\{p \in \mathbb{R}^{n+2}: w(p)=w\left(p_{0}\right)\right\}
$$

$$
\tilde{A}^{w}\left(p_{0}\right)= \pm \frac{D^{2} w\left(p_{0}\right)}{|D w|\left(p_{0}\right)} .
$$

In our case $\mathcal{S}_{\varepsilon}=\left\{p \in \mathbb{R}^{n+2}: w(p)=\varepsilon\right\}$ is such a level set for $w:=\frac{\rho}{f_{0}}$, where we write for short $\rho \equiv \operatorname{dist}_{\partial \mathcal{O}}$.

Observe that the second term on the right-hand side in

$$
\bar{\nabla} w=\frac{1}{f_{0}} \bar{\nabla} \rho-\frac{\rho}{f_{0}^{2}} \nabla f_{0}=\frac{1}{f_{0}} \nu_{\mathcal{O}}-\frac{\rho}{f_{0}^{2}} \nabla f_{0}
$$

is small if $\varepsilon$ and thus $\rho\left(p_{0}\right)$ is small, more precisely,

$$
\left|\bar{\nabla} w-\frac{1}{f_{0}} \nu_{\mathcal{O}}\right| \leq C \cdot \rho
$$


for a constant $C$ depending only on $L$ and the choice of $f_{0}$.

In particular, the normal to $\mathcal{S}_{\varepsilon}$ at $p_{0}$ is given by

$$
\nu_{\mathcal{S}_{\varepsilon}}\left(p_{0}\right)=\nu_{\mathcal{O}}\left(p_{0}\right)+\rho\left(p_{0}\right) \cdot \xi
$$

for some vector $\xi$ whose length is again bounded in terms of the function $f_{0}$ and $L$.

Similarly, we can adjust the orthonormal basis $\left(e_{a}\right)$ of the tangent space to $\partial \mathcal{O}_{\delta}$, $\delta=\rho\left(p_{0}\right)=\varepsilon \cdot f_{0}\left(p_{0}\right)$, to give an orthonormal basis $e_{a}+\rho \cdot \xi_{a}$ of $T_{p_{0}} \mathcal{S}_{\varepsilon}$, again with $\left|\xi_{a}\right| \leq C$ as above.

To prove the claim we now show that

$$
\left|A^{\mathcal{S}_{\varepsilon}}\left(p_{0}\right)\left(e_{a}+\rho \cdot \xi_{a}, e_{b}+\rho \cdot \xi_{b}\right)\right| \leq\left|A^{\mathcal{O}}\left(p_{0}\right)\left(e_{a}, e_{b}\right)\right|+C \rho \cdot\left(1+\left|A^{\mathcal{O}}\left(p_{0}\right)\right|\right) .
$$

For this we first observe that the final term of

$$
D^{2} w=\frac{1}{f_{0}} D^{2} \rho-\frac{1}{f_{0}^{2}}\left(D \rho \otimes D f_{0}+D f_{0} \otimes D \rho\right)-\frac{\rho}{f_{0}^{3}}\left(f_{0} \cdot D^{2} f_{0}-2 D f_{0} \otimes D f_{0}\right),
$$

which contains $\rho$ itself rather than a derivative of it, must be small if $\varepsilon$ is small.

As $e_{a}$ is orthogonal to $\nu_{\mathcal{O}}$, we have $D \rho\left(e_{a}\right)=0$, so evaluating the second term for the basis $\left(e_{a}+\rho \xi_{a}\right)$ of $T_{p_{0}} \mathcal{S}_{\varepsilon}$ gives also just a contribution of order $C \rho$, again with $C$ depending only on $f_{0}$ and $L$, in particular independent of the obstacle since $|D \rho|=1$.

Finally observe that the restriction of $D^{2} \rho$ to $T_{p_{0}} \partial \mathcal{O}_{\delta}$ is nothing else than the second fundamental form $A^{\mathcal{O}}$ of the level sets of the obstacle while $D^{2} \rho\left(\nu_{\mathcal{O}}, \cdot\right)$ vanishes.

Combined with (6.3) we thus find that for $\varepsilon>0$ sufficiently small

$$
\begin{aligned}
\left|A^{\mathcal{S}_{\varepsilon}}\left(p_{0}\right)\left(e_{a}+\rho \xi_{a}, e_{b}+\rho \xi_{b}\right)\right| & \leq \frac{1}{|D w|}\left[\frac{1}{f_{0}}\left|A^{\mathcal{O}}\left(p_{0}\right)\left(e_{a}, e_{b}\right)\right|+C \rho\left(1+\left|A^{\mathcal{O}}\left(p_{0}\right)\right|\right)\right] \\
& \leq\left|A^{\mathcal{O}}\left(p_{0}\right)\left(e_{a}, e_{b}\right)\right|+C \varepsilon\left(1+\left|A^{\mathcal{O}}\left(p_{0}\right)\right|\right)
\end{aligned}
$$

with constants that depend only on $L$ and the function $f_{0}$. The first claim of the lemma immediately follows.

To obtain the second claim, we recall the well-known fact, see e.g. [10, Lemma 14.17], that in a tubular neighbourhood of $\partial \mathcal{O}$ one can express the principal curvatures of the level sets $\partial \mathcal{O}_{\delta}$ in terms of $\delta$ and the principal curvatures of $\partial \mathcal{O}$. In particular, there is a constant $\varepsilon_{2}>0$ depending only on $\sup _{\partial \mathcal{O} \cap\left\{x^{n+2}>L-1\right\}}\left|A^{\mathcal{O}}\right|$ so that for any $p$ with $\left|\operatorname{dist}_{\partial \mathcal{O}}(p)\right| \leq \varepsilon_{2}$, we have $\left|A^{\mathcal{O}}(p)\right| \leq \frac{3}{2} \sup _{\partial \mathcal{O} \cap B_{1}(p)}\left|A^{\mathcal{O}}\right|$. Reducing $\varepsilon_{2}$ if necessary and combining this with the estimate proven above immediately yields the second claim.

Proof of Lemma 6.1. As we work on a compact space-time domain, here $\overline{B_{R}(0)} \times$ $[0, T]$, standard versions of the maximum principle apply. In particular, if $u$ is a solution of

$$
\dot{u}=\sqrt{1+|D u|^{2}} \cdot\left(\operatorname{div}\left(\frac{D u}{\sqrt{1+|D u|^{2}}}\right)+\alpha_{\varepsilon}\right)
$$

and $v$ is a subsolution of this equation, be it in the classical sense that $v \in C^{2}$ and $\dot{v} \leq \sqrt{1+|D v|^{2}} \cdot\left(\operatorname{div}\left(\frac{D v}{\sqrt{1+|D v|^{2}}}\right)+\alpha_{\varepsilon}\right)$, or in the viscosity sense, with $v \leq u$ on the parabolic boundary of our domain, then we have $v \leq u$ on all of $\overline{B_{R}(0)} \times[0, T]$. We may apply this to derive the lemma as follows:

(i) As $\alpha_{\varepsilon} \geq 0$, any constant function is a subsolution of (6.4). In particular, the constant $L$ acts as a lower barrier for the solution $u$ of (6.1). 
(ii) We choose a monotonically nonincreasing function $f_{0} \in C_{\mathrm{loc}}^{2}\left(\mathbb{R}, \mathbb{R}^{+}\right)$such that

$$
f_{0}(s) \geq-\beta^{-1}\left(2 \sqrt{n} \cdot\left[\sup _{\left\{x^{n+2} \geq s-1\right\} \cap \partial \mathcal{O}}\left|A^{\mathcal{O}}\right|+1\right]\right)
$$

and consider as comparison hypersurface $\mathcal{S}_{\varepsilon}$ for $\varepsilon \in\left(0, \varepsilon_{2}\right)$ as in Lemma 6.2 (for $f_{0}(p) \equiv f_{0}\left(p^{n+2}\right)$ ). Given an arbitrary point $p \in \mathcal{S}_{\varepsilon} \cap\left\{x^{n+2} \geq L-1\right\}$, we observe that

$$
\begin{aligned}
\left|H^{\mathcal{S}_{\varepsilon}}(p)\right| & \leq \sqrt{n}\left|A^{\mathcal{S}_{\varepsilon}}(p)\right| \leq 2 \sqrt{n} \cdot\left[\sup _{\partial \mathcal{O} \cap\left\{x^{n+2} \geq p^{n+2}-1\right\}}\left|A^{\mathcal{O}}\right|+1\right] \\
& \leq \beta\left(-f_{0}\left(p^{n+2}\right)\right)=\beta\left(\frac{\operatorname{dist}_{\mathcal{O O}}(p)}{\varepsilon}\right)=\alpha_{\varepsilon}(p) .
\end{aligned}
$$

Consequently, writing the stationary hypersurface $\mathcal{S}_{\varepsilon} \cap\left\{x^{n+2}>L-1\right\}$ as graph of a function $u_{S_{\varepsilon}}: U_{S_{\varepsilon}} \rightarrow \mathbb{R}$ over a subset $U_{S_{\varepsilon}} \subset B_{R}(0)$, we know that also $u_{S_{\varepsilon}}$ is a subsolution of (6.4).

(iii) As $u_{S_{\varepsilon}}$ is not defined on the whole of $\overline{B_{R}(0)}$ we first cut off $u_{S_{\varepsilon}}$ to $\max \left\{u_{S_{\varepsilon}}, L\right\}$ on its domain of definition $U_{S_{\varepsilon}}$ and observe that this function is equal to $L$ in a neighbourhood of $\partial U_{S_{\varepsilon}}$ so can be continuously extended by $L$ to all of $\overline{B_{R}(0)}$. As the maximum of two subsolutions is again a subsolution, now in the viscosity sense, we thus obtain that graph $u$ remains above both $\mathcal{S}_{\varepsilon}$ and the plane $\left\{x^{n+2}=L\right\}$ for all times and $(6.2)$ is valid with $C_{0}(\ell)=f_{0}(\ell-1)$.

Based on Lemma 6.1, we will henceforth assume

Assumption 6.3 (Standard assumption on $\varepsilon$ ). Given a number $L \in \mathbb{R}$ and an initial hypersurface $M_{0}$ (disjoint from the obstacle) contained in $\left\{x^{n+2} \geq L\right\}$, we consider the evolution equation (3.2) only for values of $\varepsilon \in\left(0, \varepsilon_{0}(L)\right)$, the number given by Lemma 6.1.

As a consequence of Lemma 6.1 and its proof, we get the following more general bounds on the penetration depth of solutions to (3.2)

Corollary 6.4. Let $\mathcal{O}$ be an obstacle as in Definition 2.1 which we furthermore assume to be of class $C^{2}$ and let $\ell>-\infty$ be any number. Then there exist $K<\infty$ and $C_{0}>0$ such that the following holds true.

Let $\left(M_{t}\right)_{t}$ be a smooth solution of (3.2) (with $\varepsilon$ satisfying the standard assumption) which is initially disjoint from the obstacle. Then $\operatorname{dist}_{\partial \mathcal{O}}(p) \geq-C_{0} \varepsilon$ and

$$
\alpha_{\varepsilon}(p)+\left|A^{\mathcal{O}}\right|(p)+v^{\mathcal{O}}(p) \leq K
$$

for any $p \in M_{t} \cap \mathcal{O} \cap\left\{x^{n+2} \geq \ell\right\}$ and any $t \geq 0$.

We remark that the above constant $K$ depends only on local $C^{2}$-bounds of the obstacle. In particular, while in Definition 2.1 the assumed regularity of the obstacle is only $C^{1,1}$ and not $C^{2}$, we can and will approximate such obstacles by smooth obstacles with locally bounded $C^{2}$-norm, so Corollary 6.4 will still apply with constants depending only on the local $C^{1,1}$-norms of the original obstacle $\mathcal{O}$.

In the following Sections 7 and 8, we shall derive a priori estimates for solutions of (3.2) in such halfspaces $\left\{x^{n+2} \geq \ell\right\}$. We will apply these a priori estimates later on in Section 9 to control the evolution of the approximate solutions constructed in Proposition 9.1 and will always choose $\ell$ large enough so that the following holds:

Assumption 6.5 (Assumptions for a priori estimates in $\left\{x^{n+2} \geq \ell\right\}$ ). We consider solutions $\left(M_{t}\right)_{t}$ of (3.2) with the following properties: For some $a>0$

(i) each $M_{t} \cap\left\{x^{n+2}>\ell-a\right\}, t \geq 0$, is a graphical, smooth submanifold without boundary and

(ii) each $M_{t} \cap\left\{x^{n+2} \geq \ell-a\right\}$ is compact. 


\section{7. $C^{1}$-ESTIMATES FOR THE GRAPHICAL FLOW: GRADIENT FUNCTION}

We combine the evolution equation for the gradient function given in Lemma 5.2 with the key observation concerning the additional term $\left\langle\nabla \alpha_{\varepsilon}, e_{n+2}\right\rangle$ made in Remark 5.3 and a localisation argument to prove

Proposition 7.1. Let $\ell \in \mathbb{R}$ and let $\left(M_{t}\right)_{t}$ be a solution of $(3.2)$, with $\varepsilon$ as in Assumption 6.3, such that Assumption 6.5 is satisfied. Then the gradient function is controlled by

$$
(U-\ell)^{2} \cdot v \leq \sup _{M_{0} \cap\left\{x^{n+2} \geq \ell\right\}}(U-\ell)^{2} \cdot v+C(\ell),
$$

for all times and in all points with height $U \geq \ell$. Here $C(\ell)$ depends only on $\max _{M_{0}} U-\ell$ and the bounds for $v_{\mathcal{O}}$ and $\alpha_{\varepsilon}$ from Corollary 6.4 .

The proof of this proposition is based on the maximum principle. It is important to observe that both in the present proof, and in similar arguments carried out in Section 8 , we only need to apply the maximum principle on the compact domains $\{(x, t): u(x, t) \geq \ell, t \in[0, T]\}$ as our quantities are constructed so that they are zero on the spatial boundary of this domain, on a neighbourhood of which the hypersurface is smooth and graphical, compare Assumption 6.5.

Proof. We may assume without loss of generality that $\ell=0$. We want to apply the maximum principle to the function

$$
w:=U^{2} v
$$

and obtain by direct computation

$$
\begin{aligned}
\dot{w}-\Delta w= & 2 U v(\dot{U}-\Delta U)+U^{2}(\dot{v}-\Delta v)-2 v|\nabla U|^{2}-4 U\langle\nabla U, \nabla v\rangle \\
= & 2 U v \frac{\alpha_{\varepsilon}}{v}+U^{2}\left(-|A|^{2} v-\frac{2}{v}|\nabla v|^{2}+v^{2}\left\langle\nabla \alpha_{\varepsilon}, e_{n+2}\right\rangle\right) \\
& -2 v|\nabla U|^{2}-4 U\langle\nabla U, \nabla v\rangle .
\end{aligned}
$$

At a spatial maximum of $w$, we obtain

$$
\begin{aligned}
0= & 2 U v \nabla U+U^{2} \nabla v \\
\dot{w}-\Delta w= & 2 U \alpha_{\varepsilon}-U^{2}|A|^{2} v-\frac{2}{v}|\nabla v|^{2} U^{2}+v^{2} U^{2}\left\langle\nabla \alpha_{\varepsilon}, e_{n+2}\right\rangle \\
& -2 v|\nabla U|^{2}+2 U^{2} \frac{1}{v}|\nabla v|^{2} \\
\leq & 2 U \alpha_{\varepsilon}+v^{2} U^{2}\left\langle\nabla \alpha_{\varepsilon}, e_{n+2}\right\rangle-2 v\left(1-\frac{1}{v^{2}}\right)
\end{aligned}
$$

where we have used, setting $\eta=e_{n+2}$ and observing $|\eta|=1$, that

$$
|\nabla U|^{2}=\eta_{\gamma} \nabla_{i} F^{\gamma} g^{i j} \nabla_{j} F^{\zeta} \eta_{\zeta}=\eta_{\gamma}\left(\delta^{\gamma \zeta}-\nu^{\gamma} \nu^{\zeta}\right) \eta_{\zeta}=|\eta|^{2}-\langle\nu, \eta\rangle^{2}=1-\frac{1}{v^{2}}
$$

If $w$ is large, $v$ is also large since the hyperplane $\left\{x^{n+2}=\sup u_{0}\right\}$ is a stationary solution of the flow and hence acts as an upper barrier. In this situation, $\left\langle\nabla \alpha_{\varepsilon}, e_{n+2}\right\rangle \leq 0$ according to Remark 5.3. The term $2 U \alpha_{\varepsilon}$ is uniformly bounded and can be absorbed as $-2 v+2 / v \leq-v$ for $v \geq 2$. Hence the claimed inequality follows from the maximum principle as $w$ vanishes at height $\ell$. 


\section{Controlling the SECOND Fundamental Form}

In this section we analyse the evolution of the second fundamental form under the flow (3.2). According to (5.11), we have

$$
\begin{aligned}
\left(\frac{d}{d t}-\Delta\right)|A|^{2}= & -|\nabla A|^{2}+2|A|^{4}-2 \alpha_{\varepsilon} A_{i}^{k} A_{j}^{i} A_{k}^{j} \\
& -2 \beta_{\varepsilon}^{\prime \prime}\left\langle\nu_{\mathcal{O}}, \nabla_{i} F\right\rangle \cdot\left\langle\nu_{\mathcal{O}}, \nabla_{j} F\right\rangle A^{i j}-2 \beta_{\varepsilon}^{\prime} \tilde{A}_{i j}^{\mathcal{O}} A^{i j}+2 \beta_{\varepsilon}^{\prime}\left\langle\nu_{,} \nu_{\mathcal{O}}\right\rangle|A|^{2},
\end{aligned}
$$

where the first two terms agree with the evolution equation for standard mean curvature flow.

The additional terms are all supported on the obstacle though with vastly different behaviour as $\varepsilon \searrow 0$, depending on whether or not the term contains derivatives of the penalty function $\alpha_{\varepsilon}$.

Namely, as $\alpha_{\varepsilon}$ is bounded uniformly in time in every halfspace $\left\{x^{n+2} \geq \ell\right\}$, see Section 6 , the term $-2 \alpha_{\varepsilon} A_{i}^{k} A_{j}^{i} A_{k}^{j}$ will be dominated by $|A|^{4}$ in points where the second fundamental form is large and as such will not play an important role, no matter how small $\varepsilon$ is.

Conversely, all other terms contain derivatives of $\alpha_{\varepsilon}$ and can thus be of order $\varepsilon^{-1}$ (for first order derivatives as occurring in the last two terms in (8.1)) or even $\varepsilon^{-2}$ (for the other additional term) in points of the obstacle that can a priori be reached by the evolving hypersurface, compare Section 6 .

These terms cannot be expected to have a sign so that we need to construct a modified second fundamental form quantity in order to be able to apply the maximum principle.

This construction is done in three steps, first replacing $|A|^{2}$ with a quantity $f$ whose evolution equation resembles more closely the one of $|A|^{2}$ for standard mean curvature flow, then, similarly to [5] further modifying this to obtain a quantity $G$ for which $\left(\frac{d}{d t}-\Delta\right) G$ is negative for large values of $G$ and controlled gradient and then finally by localising in space-time.

We first prove

Lemma 8.1. For any $\eta \in(0,1)$ and $\ell>-\infty$, there exists a constant $\gamma_{0} \in(0,1]$, so that to any $\gamma \in\left(0, \gamma_{0}\right]$, we can choose $1 \leq \bar{F}=\bar{F}(\eta, \ell, \gamma)<\infty$, such that the following holds true.

Let $\left(M_{t}\right)_{t}$ be a smooth solution of the flow (3.2) (for $\varepsilon$ in the range $\left(0, \varepsilon_{1}\right)$ as discussed in Assumption 6.3). Then the inequality

$$
\begin{aligned}
e^{-\gamma \alpha_{\varepsilon}}\left(\frac{d}{d t}-\Delta_{M}\right)\left(e^{\gamma \alpha_{\varepsilon}}|A|^{2}\right) \leq & -(2-\eta)|\nabla A|^{2}+(2+\eta)|A|^{4} \\
& -\left|\beta_{\varepsilon}^{\prime}\right| \cdot\left\langle\nu, \nu_{\mathcal{O}}\right\rangle_{+}|A|^{2}-\frac{\gamma}{4} \beta_{\varepsilon}^{\prime \prime}|A|^{2}\left|P_{T M} \nu_{\mathcal{O}}\right|^{2}
\end{aligned}
$$

holds in every point $p \in M_{t} \cap\left\{x^{n+2} \geq \ell\right\}$ in which

$$
|A| \geq \bar{F} \text {. }
$$

Recall that $\alpha_{\varepsilon}$ is uniformly bounded in points $p \in M_{t} \cap\left\{x^{n+2} \geq \ell\right\}$, see Corollary 6.4. Hence in points where $e^{\gamma \alpha_{\varepsilon}}|A|^{2}$ is large, $|A|$ is also large and the estimate above applies. Therefore inequalities as in Lemma 8.1, valid only where $|A|$ is large and thus of a much simpler form than the general evolution equation, are suitable to derive upper bounds on the second fundamental form.

We remark that while the present lemma makes no use of the $C^{1}$-bounds on the evolving hypersurface derived earlier, such bounds will be crucial in the following lemma.

Lemma 8.2. For any numbers $M<\infty$ and $\ell>-\infty$, there exist numbers $\gamma, k>0$ as well as $\bar{F}<\infty$, such that the following holds. Let $\left(M_{t}\right)_{t}$ be a smooth solution of 
(3.2) for some $\varepsilon \in\left(0, \varepsilon_{1}\right)$ as in Assumption 6.3 and set

$$
G:=h\left(v^{2}\right) \cdot e^{\gamma \alpha_{\varepsilon}} \cdot|A|^{2}, \text { where } h(y)=y \cdot e^{k y} .
$$

Then

$$
\begin{aligned}
\left(\frac{d}{d t}-\Delta\right) G+\frac{1}{h}\langle\nabla h, \nabla G\rangle \leq & -\frac{k}{8}\left[h e^{\gamma \alpha_{\varepsilon}}|\nabla A|^{2}+G|A|^{2}+G|\nabla v|^{2}\right] \\
& -\left[\frac{\gamma}{8} \beta_{\varepsilon}^{\prime \prime}\left|P_{T M} \nu_{\mathcal{O}}\right|^{2}+\frac{1}{2}\left|\beta_{\varepsilon}^{\prime}\right|\left\langle\nu, \nu_{\mathcal{O}}\right\rangle_{+}\right] \cdot G
\end{aligned}
$$

holds in every point $p \in M_{t} \cap\left\{x^{n+2} \geq \ell\right\}$, where $|A|$ is large and the gradient function $v$ of $M_{t}$ is bounded, namely

$$
|A(p)| \geq \bar{F}, \text { while } v(p) \leq M
$$

Proof of Lemma 8.1. Let $\eta>0$ and $\ell>-\infty$ be given. Let $K$ be as in Corollary 6.4 and let $\left(M_{t}\right)_{t}$ be a solution of the flow (3.2) for some number $\varepsilon \in\left(0, \varepsilon_{1}\right)$ as in Assumption 6.3. Then for $\gamma$ in a range $\left(0, \gamma_{0}\right)$ to be determined later, we set

$$
f=f_{\gamma}=e^{\gamma \alpha_{\varepsilon}}|A|^{2}
$$

and compute, using (5.11) and (5.12),

$$
\begin{aligned}
e^{-\gamma \alpha_{\varepsilon}\left[\left(\frac{d}{d t}-\Delta\right) f\right]=} & \left(\frac{d}{d t}-\Delta\right)|A|^{2}-2 \gamma\left\langle\nabla \alpha_{\varepsilon}, \nabla|A|^{2}\right\rangle \\
& +\gamma|A|^{2} \cdot\left(\frac{d}{d t}-\Delta\right) \alpha_{\varepsilon}-\gamma^{2}\left|\nabla \alpha_{\varepsilon}\right|^{2}|A|^{2} \\
= & -2|\nabla A|^{2}+2|A|^{4}-2 \alpha_{\varepsilon} A_{i}^{k} A_{j}^{i} A_{k}^{j} \\
& -\beta_{\varepsilon}^{\prime \prime}\left\langle\nu_{\mathcal{O}}, \nabla_{i} F\right\rangle \cdot\left\langle\nu_{\mathcal{O}}, \nabla_{j} F\right\rangle A^{i j}-2 \beta_{\varepsilon}^{\prime} \tilde{A}_{i j}^{\mathcal{O}} A^{i j} \\
& +2 \beta_{\varepsilon}^{\prime}\left\langle\nu, \nu_{\mathcal{O}}\right\rangle|A|^{2}-2 \gamma \beta_{\varepsilon}^{\prime}\left\langle P_{T M} \nu_{\mathcal{O}}, \nabla|A|^{2}\right\rangle \\
& +\gamma\left[\beta_{\varepsilon}^{\prime}\left\langle\nu_{\mathcal{O}}, \alpha_{\varepsilon} \nu\right\rangle-\beta_{\varepsilon}^{\prime \prime}\left|P_{T M} \nu_{\mathcal{O}}\right|^{2}-\beta_{\varepsilon}^{\prime} \tilde{A}_{i j}^{\mathcal{O}} g^{i j}\right]|A|^{2} \\
& -\gamma^{2}\left|\beta_{\varepsilon}^{\prime}\right|^{2}\left|P_{T M} \nu_{\mathcal{O}}\right|^{2}|A|^{2} .
\end{aligned}
$$

Dropping the last, obviously non-positive term and using Young's inequality as well as Kato's inequality $|\nabla| A|| \leq|\nabla A|$, we obtain

$$
\begin{aligned}
e^{-\gamma \alpha_{\varepsilon}\left[\left(\frac{d}{d t}-\Delta\right) f\right] \leq} & -(2-\eta)|\nabla A|^{2}+\left(2+\frac{\eta}{2}\right)|A|^{4}+\frac{2}{\eta}|A|^{2} \alpha_{\varepsilon}{ }^{2} \\
& +C\left|\beta_{\varepsilon}^{\prime}\right| \cdot\left|A^{\mathcal{O}}\right| \cdot\left(\gamma|A|^{2}+|A|\right) \\
& -\left|P_{T M} \nu_{\mathcal{O}}\right|^{2} \cdot\left[\beta_{\varepsilon}^{\prime \prime}\left(\gamma|A|^{2}-|A|\right)-\frac{4 \gamma^{2}}{\eta}\left|\beta_{\varepsilon}^{\prime}\right|^{2}|A|^{2}\right] \\
& -\left\langle\nu_{\nu_{\mathcal{O}}}\right\rangle\left(2+\gamma \alpha_{\varepsilon}\right)\left|\beta_{\varepsilon}^{\prime}\right||A|^{2} .
\end{aligned}
$$

To rewrite this expression in the form

$$
\begin{aligned}
e^{-\gamma \alpha_{\varepsilon}\left[\left(\frac{d}{d t}-\Delta\right) f\right] \leq} & -(2-\eta)|\nabla A|^{2}+\left(2+\frac{\eta}{2}\right)|A|^{4}+\frac{2}{\eta}|A|^{2} \alpha_{\varepsilon}{ }^{2} \\
& -\left|P_{T M \nu_{\mathcal{O}}}\right|^{2} \cdot T_{1}-\left\langle\nu, \nu_{\mathcal{O}}\right\rangle \cdot T_{2},
\end{aligned}
$$

we then use that

$$
1=\left|\nu_{\mathcal{O}}\right|^{2}=\left|P_{T M} \nu_{\mathcal{O}}\right|^{2}+\left\langle\nu, \nu_{\mathcal{O}}\right\rangle^{2}
$$


to split the term on the second line of (8.3) into suitable multiples of $\left|P_{T M} \nu_{\mathcal{O}}\right|^{2}$ and of $\left\langle\nu, \nu_{\mathcal{O}}\right\rangle$ and find that (8.4) is valid for

$$
\begin{aligned}
T_{1}:= & {\left[\gamma \beta_{\varepsilon}^{\prime \prime}-\frac{4 \gamma^{2}}{\eta}\left|\beta_{\varepsilon}^{\prime}\right|^{2}-C \gamma\left|\beta_{\varepsilon}^{\prime}\right|\left|A^{\mathcal{O}}\right|\right] \cdot|A|^{2}-\left[C\left|\beta_{\varepsilon}^{\prime}\right|\left|A^{\mathcal{O}}\right|+\beta_{\varepsilon}^{\prime \prime}\right] \cdot|A| } \\
\geq & \gamma \cdot\left[\beta_{\varepsilon}^{\prime \prime}-\gamma\left(4 \eta^{-1}+1\right)\left|\beta_{\varepsilon}^{\prime}\right|^{2}\right]|A|^{2}-\left[\beta_{\varepsilon}^{\prime \prime}+\left|\beta_{\varepsilon}^{\prime}\right|^{2}\right] \cdot|A| \\
& -C\left|A^{\mathcal{O}}\right|^{2}\left(|A|^{2}+1\right)
\end{aligned}
$$

and

$$
T_{2}=\left(2+\gamma \alpha_{\varepsilon}\right)\left|\beta_{\varepsilon}^{\prime}\right||A|^{2}-C\left\langle\nu, \nu_{\mathcal{O}}\right\rangle\left|\beta_{\varepsilon}^{\prime}\right| \cdot\left|A^{\mathcal{O}}\right| \cdot\left(\gamma|A|^{2}+|A|\right),
$$

$C=C(n)$ some universal constants.

We will first show that the dominating term in $T_{1}$ is given by $\gamma \beta_{\varepsilon}^{\prime \prime}|A|^{2}>0$, so that we obtain a negative contribution to the right-hand side of (8.4) scaling as $\varepsilon^{-2}$ in points where $P_{T M} \nu_{\mathcal{O}}$ is non-zero, i. e. in points where the tangent plane of the evolving hypersurface and the obstacle do not coincide.

Conversely, as both the obstacle and the evolving hypersurface are graphical, it is precisely in points where the two tangent planes coincide that $\left\langle\nu, \nu_{\mathcal{O}}\right\rangle$ is maximal, i. e. equal to one, so, as we shall see, we again get a large negative contribution to the right hand side of (8.4) now coming from the dominating term $2\left|\beta_{\varepsilon}^{\prime}\right||A|^{2}$ of $T_{2}$.

To begin with we show

Claim: Given any $\eta>0$ there exists $\gamma_{0}>0$ such that for any $\gamma \in\left(0, \gamma_{0}\right)$ there is a number $\bar{F}$ such that

$$
T_{1} \geq\left(\frac{\gamma}{2} \beta_{\varepsilon}^{\prime \prime}-C \cdot K^{2}\right) \cdot|A|^{2}
$$

in every point $p \in M_{t} \cap\left\{x^{n+2} \geq \ell\right\}$ in which $|A| \geq \bar{F}$. Here $C$ is a universal constant and $K=K(\ell)$ is the number given in Corollary 6.4.

To prove this claim, we first recall from Corollary 6.4 that $\operatorname{dist}_{\partial \mathcal{O}}(p) \geq-c_{0} \cdot \varepsilon$, $c_{0}=c_{0}(\ell)$. Thus $\beta_{\varepsilon}$ and its derivatives need to be evaluated only for arguments contained in an interval $\left[-c_{0} \varepsilon, \infty\right)$ where

$$
\frac{\left(\beta_{\varepsilon}^{\prime}\right)^{2}}{\beta_{\varepsilon}^{\prime \prime}} \leq \sup _{\left[-c_{0}, 0\right]} \frac{\left(\beta^{\prime}\right)^{2}}{\beta^{\prime \prime}} \leq C_{1}
$$

is bounded by a constant depending only on $c_{0}$ (and thus $\ell$ ) and the function $\beta$, which we had chosen so that $\beta^{\prime \prime \prime} \leq 0$.

In points where $|A|$ is large, $|A| \geq \bar{F}$ for $\bar{F} \geq 1$ still to be determined, we thus get

(8.6) $T_{1} \geq \gamma \beta_{\varepsilon}^{\prime \prime} \cdot\left[1-\gamma C_{1}\left(4 \eta^{-1}+1\right)-(\gamma \bar{F})^{-1}\left(1+C_{1}\right)\right]|A|^{2}-C\left|A^{\mathcal{O}}\right|^{2}|A|^{2}$.

Choosing $\gamma_{0} \in(0,1)$ small enough so that $\gamma_{0} C_{1}\left(4 \eta^{-1}+1\right) \leq \frac{1}{4}$, and then, for each $\gamma \in\left(0, \gamma_{0}\right)$, selecting a number $\bar{F}$ large enough so that $(\gamma \bar{F})^{-1}\left(1+C_{1}\right) \leq \frac{1}{4}$, we thus find as claimed that

$$
T_{1} \geq \frac{\gamma}{2} \beta_{\varepsilon}^{\prime \prime}|A|^{2}-C \cdot K^{2}|A|^{2}
$$

where we use Corollary 6.4 to deal with the last term in (8.6).

To analyse $T_{2}$, we first observe that

$$
\begin{aligned}
\left.\left|T_{2}-2\right| \beta_{\varepsilon}^{\prime}|| A\right|^{2} \mid & \leq \gamma \alpha_{\varepsilon}\left|\beta_{\varepsilon}^{\prime}\right||A|^{2}+C\left|\beta_{\varepsilon}^{\prime}\right| \cdot\left|A^{\mathcal{O}}\right| \cdot|A|^{2} \cdot\left(\gamma+|A|^{-1}\right) \\
& \leq \gamma_{0} K\left|\beta_{\varepsilon}^{\prime}\right||A|^{2}+C \gamma_{0} K\left|\beta_{\varepsilon}^{\prime}\right||A|^{2}+C \bar{F}^{-1} K\left|\beta_{\varepsilon}^{\prime}\right||A|^{2}
\end{aligned}
$$




$$
\leq C K\left(\gamma_{0}+\bar{F}^{-1}\right)\left|\beta_{\varepsilon}^{\prime}\right||A|^{2},
$$

since we only need to consider points with $|A| \geq \bar{F}$. After possibly reducing $\gamma_{0}$ and increasing $\bar{F}$, we thus obtain

$$
3\left|\beta_{\varepsilon}^{\prime}\right||A|^{2} \geq T_{2} \geq\left|\beta_{\varepsilon}^{\prime}\right||A|^{2} .
$$

Remark that these expressions only scale as $\varepsilon^{-1}$ and not as $\varepsilon^{-2}$ like the leading order term of $T_{1}$.

This difference is crucial since we cannot expect to control the sign of $\left\langle\nu, \nu_{\mathcal{O}}\right\rangle$ and will thus need to rely on the contribution of $T_{1}$ to (8.4) in points where this inner product is negative. While not necessarily positive, we observe that since both the obstacle and the evolving hypersurface are graphical, this inner product is bounded away from -1 . Namely writing $\nu_{\mathcal{O}}=\left\langle\nu_{\mathcal{O}}, e_{n+2}\right\rangle e_{n+2}+P_{\mathbb{R}^{n+1}} \nu_{\mathcal{O}}$, where $P_{\mathbb{R}^{n+1}} \nu_{\mathcal{O}}$ is the orthogonal projection of $\nu_{\mathcal{O}}$ onto $\mathbb{R}^{n+1} \times\{0\}$, we find

$$
\begin{aligned}
\left\langle\nu_{\mathcal{O}}, \nu\right\rangle & =\left\langle\left\langle\nu_{\mathcal{O}}, e_{n+2}\right\rangle e_{n+2}+P_{\mathbb{R}^{n+1}} \nu_{\mathcal{O}}, \nu\right\rangle=\left\langle\nu_{\mathcal{O}}, e_{n+2}\right\rangle \cdot\left\langle\nu, e_{n+2}\right\rangle+\left\langle P_{\mathbb{R}^{n+1}} \nu_{\mathcal{O}}, \nu\right\rangle \\
& \geq\left(v \cdot v_{\mathcal{O}}\right)^{-1}-\left|P_{\mathbb{R}^{n+1}} \nu_{\mathcal{O}}\right| \geq-\left|P_{\mathbb{R}^{n+1}} \nu_{\mathcal{O}}\right|=-\sqrt{1-\left\langle\nu_{\mathcal{O}}, e_{n+2}\right\rangle^{2}} \\
& \geq-\sqrt{1-K^{-2}}
\end{aligned}
$$

with the last inequality due to Corollary 6.4.

In points where $\left\langle\nu, \nu_{\mathcal{O}}\right\rangle<0$, we may thus bound

$$
\left|P_{T M} \nu_{\mathcal{O}}\right|^{2}=1-\left|\left\langle\nu, \nu_{\mathcal{O}}\right\rangle\right|^{2} \geq K^{-2}
$$

which in turn gives

$$
\left\langle\nu_{\mathcal{O}}, \nu\right\rangle=\left\langle\nu_{\mathcal{O}}, \nu\right\rangle_{+}-\left\langle\nu_{\mathcal{O}}, \nu\right\rangle_{-} \geq\left\langle\nu_{\mathcal{O}}, \nu\right\rangle_{+}-K^{2}\left|P_{T M} \nu_{\mathcal{O}}\right|^{2} .
$$

Considering points $p \in M_{t} \cap\left\{x^{n+2} \geq \ell\right\}$ with $|A| \geq \bar{F}$, we can thus conclude from (8.7) and (8.8) that the estimate

$$
\left|P_{T M} \nu_{\mathcal{O}}\right|^{2} T_{1}+\left\langle\nu, \nu_{\mathcal{O}}\right\rangle T_{2} \geq\left|P_{T M} \nu_{\mathcal{O}}\right|^{2} \cdot\left(\frac{\gamma}{4} \beta_{\varepsilon}^{\prime \prime}-C\right)|A|^{2}+\left\langle\nu, \nu_{\mathcal{O}}\right\rangle_{+}\left|\beta_{\varepsilon}^{\prime}\right||A|^{2}
$$

holds with a constant $C=C(K)$, at least if $\left\langle\nu, \nu_{\mathcal{O}}\right\rangle \geq 0$. On the other hand, if $\left\langle\nu, \nu_{\mathcal{O}}\right\rangle<0$, we can combine (8.7) and (8.8) with (8.9) to conclude that

$$
\begin{aligned}
\left|P_{T M} \nu_{\mathcal{O}}\right|^{2} T_{1}+\left\langle\nu, \nu_{\mathcal{O}},\right\rangle T_{2} & \geq\left|P_{T M} \nu_{\mathcal{O}}\right|^{2} \cdot\left(\frac{\gamma}{2} \beta_{\varepsilon}^{\prime \prime}-3\left|\beta_{\varepsilon}^{\prime}\right| K^{2}\right) \cdot|A|^{2}-C \cdot|A|^{2} \\
& \geq\left|P_{T M} \nu_{\mathcal{O}}\right|^{2} \cdot\left(\frac{\gamma}{2} \beta_{\varepsilon}^{\prime \prime}-\gamma^{2}\left|\beta_{\varepsilon}^{\prime}\right|^{2}\right)|A|^{2}-C\left(1+\gamma^{-2}\right)|A|^{2} \\
& \geq\left|P_{T M} \nu_{\mathcal{O}}\right|^{2} \cdot\left(\frac{\gamma}{2} \beta_{\varepsilon}^{\prime \prime}-\gamma^{2} C_{1} \beta_{\varepsilon}^{\prime \prime}\right)|A|^{2}-C(\gamma, K)|A|^{2} \\
& \geq\left|P_{T M} \nu_{\mathcal{O}}\right|^{2} \cdot\left[\frac{\gamma}{4} \beta_{\varepsilon}^{\prime \prime}-C(\gamma, K)\right]|A|^{2}
\end{aligned}
$$

since $\gamma_{0} C_{1} \leq \frac{1}{4}$. But in this second case $\left\langle\nu, \nu_{\mathcal{O}}\right\rangle_{+}$is zero which means that (8.10) also holds though now with a constant $C=C(\gamma, K)$. Inserting (8.10) into (8.4) thus gives

$$
\begin{aligned}
e^{-\gamma \alpha_{\varepsilon}}\left(\frac{d}{d t}-\Delta_{M}\right)\left(e^{\gamma \alpha_{\varepsilon}}|A|^{2}\right) \leq & -(2-\eta)|\nabla A|^{2}+\left(2+\frac{\eta}{2}\right)|A|^{4}+C_{2} \cdot|A|^{2} \\
& -\left|\beta_{\varepsilon}^{\prime}\right| \cdot\left\langle\nu, \nu_{\mathcal{O}}\right\rangle_{+}|A|^{2}-\frac{\gamma}{4} \beta_{\varepsilon}^{\prime \prime}|A|^{2}\left|P_{T M} \nu_{\mathcal{O}}\right|^{2}
\end{aligned}
$$

for a constant $C_{2}$ depending on $\eta, \gamma$ as well as $K$. Possibly further increasing $\bar{F}$ (which is allowed to depend on all these quantities), we can however assume that $C_{2} \leq \frac{\eta}{2}(\bar{F})^{2}$, so that we can estimate the final term on the first line by $\frac{\eta}{2}|A|^{4}$ in the points under consideration, thus obtaining the claim of the lemma. 
Proof of Lemma 8.2. Given a number $M$ and a level $\ell>-\infty$, we let $K$ be as in Corollary 6.4 and consider a smooth solution $\left(M_{t}\right)_{t}$ of the flow $(3.2), \varepsilon \in\left(0, \varepsilon_{1}\right)$ as in Assumption 6.3, in points where $M \geq v$. For a number $\eta=\eta(M, \ell)>0$ to be determined below, we let $\gamma_{0}=\gamma_{0}(\eta, \ell)>0$ be as in Lemma 8.1.

We then consider the function

$$
G=h\left(v^{2}\right) \cdot f
$$

where $f=e^{\gamma \alpha_{\varepsilon}}|A|^{2}$ is as in Lemma 8.1, with $\gamma \in\left(0, \gamma_{0}\right)$ still to be determined, and $h: \mathbb{R}^{+} \rightarrow \mathbb{R}^{+}$a nondecreasing function which we will later choose as stated in the lemma.

To begin with, we calculate

$$
\begin{aligned}
\left(\frac{d}{d t}-\Delta\right) G= & h \cdot\left(\frac{d}{d t}-\Delta\right) f+f \cdot 2 v \cdot h^{\prime} \cdot\left(\frac{d}{d t}-\Delta\right) v \\
& -\left[h^{\prime \prime} \cdot\left|\nabla\left(v^{2}\right)\right|^{2}+2 h^{\prime} \cdot|\nabla v|^{2}\right] \cdot f-2\left\langle\nabla\left(h\left(v^{2}\right)\right), \nabla f\right\rangle .
\end{aligned}
$$

Here and in the following, $h$ and its derivatives are evaluated at $y=v^{2}$ unless stated otherwise.

Let now $p \in M_{t} \cap\left\{x^{n+2} \geq \ell\right\}$ be a point where $|A| \geq \bar{F}$, the number given by Lemma 8.1. Inserting the evolution equation (5.6) of the gradient function as well as the estimate (8.2) into (8.11), we obtain

$$
\begin{aligned}
e^{-\gamma \alpha_{\varepsilon}}\left(\frac{d}{d t}-\Delta\right) G \leq h & \cdot\left[-(2-\eta)|\nabla A|^{2}+(2+\eta)|A|^{4}\right. \\
& \left.-\left|\beta_{\varepsilon}^{\prime}\right| \cdot\left\langle\nu, \nu_{\mathcal{O}}\right\rangle_{+} \cdot|A|^{2}-\frac{\gamma}{4} \beta_{\varepsilon}^{\prime \prime}|A|^{2}\left|P_{T M} \nu_{\mathcal{O}}\right|^{2}\right] \\
& +|A|^{2} \cdot 2 v \cdot h^{\prime} \cdot\left[-|A|^{2} v-\frac{2}{v}|\nabla v|^{2}+v^{2}\left\langle\nabla \alpha_{\varepsilon}, e_{n+2}\right\rangle\right] \\
& -\left(4 h^{\prime \prime} v^{2}|\nabla v|^{2}+2 h^{\prime} \cdot|\nabla v|^{2}\right) \cdot|A|^{2} \\
& -2 e^{-\gamma \alpha_{\varepsilon}}\left\langle\nabla\left(h\left(v^{2}\right)\right), \nabla f\right\rangle .
\end{aligned}
$$

We estimate the last term on the third line using Young's inequality as

$$
\begin{aligned}
|A|^{2} \cdot 2 v \cdot h^{\prime} \cdot v^{2}\left\langle\nabla \alpha_{\varepsilon}, e_{n+2}\right\rangle= & 2 v^{3}|A|^{2} h^{\prime} \beta_{\varepsilon}^{\prime}\left\langle P_{T M} \nu_{\mathcal{O}}, e_{n+2}\right\rangle \\
\leq & \gamma^{2} \frac{\left(h^{\prime}\right)^{2}}{h} \cdot v^{2}\left|\beta_{\varepsilon}^{\prime}\right|^{2}\left|P_{T M} \nu_{\mathcal{O}}\right|^{2}|A|^{2} \\
& +\gamma^{-2} v^{4} h \cdot|A|^{2} \\
\leq & \gamma^{2} \frac{\left(h^{\prime}\right)^{2}}{h} \cdot v^{2}\left|\beta_{\varepsilon}^{\prime}\right|^{2}\left|P_{T M} \nu_{\mathcal{O}}\right|^{2}|A|^{2} \\
& +\gamma^{-2} M^{4} \cdot e^{-\gamma \alpha_{\varepsilon}} \cdot G .
\end{aligned}
$$

Then, as in [5], we deal with the last term in (8.12) by writing one multiple of $e^{-\gamma \alpha_{\varepsilon}}\left\langle\nabla\left(h\left(v^{2}\right)\right), \nabla f\right\rangle$ in terms of $G=h \cdot f$ as

$$
\begin{aligned}
-e^{-\gamma \alpha_{\varepsilon}}\left\langle\nabla\left(h\left(v^{2}\right)\right), \nabla f\right\rangle & =-\frac{e^{-\gamma \alpha_{\varepsilon}}}{h} \cdot\left\langle\nabla\left(h\left(v^{2}\right)\right), \nabla G\right\rangle+\frac{e^{-\gamma \alpha_{\varepsilon}}}{h}\left|\nabla\left(h\left(v^{2}\right)\right)\right|^{2} \cdot f \\
& =-\frac{e^{-\gamma \alpha_{\varepsilon}}}{h} \cdot\left\langle\nabla\left(h\left(v^{2}\right)\right), \nabla G\right\rangle+4 \frac{\left(h^{\prime}\right)^{2}}{h} v^{2}|\nabla v|^{2}|A|^{2}
\end{aligned}
$$

while rewriting the remaining multiple as

$$
e^{-\gamma \alpha_{\varepsilon}}\left\langle\nabla\left(h\left(v^{2}\right)\right), \nabla f\right\rangle=\left\langle\nabla\left(h\left(v^{2}\right)\right), \nabla\left(|A|^{2}\right)\right\rangle+\gamma|A|^{2}\left\langle\nabla\left(h\left(v^{2}\right)\right), \nabla \alpha_{\varepsilon}\right\rangle
$$


and consequently estimating it, using Kato's and Young's inequality as well as (5.4), by

$\left|e^{-\gamma \alpha_{\varepsilon}}\left\langle\nabla\left(h\left(v^{2}\right)\right), \nabla f\right\rangle\right| \leq 4 h^{\prime} v|\nabla v| \cdot|A||\nabla A|+2 \gamma h^{\prime} \cdot v \cdot|A|^{2} \cdot \beta_{\varepsilon}^{\prime}\left\langle\nabla v, P_{T M} \nu_{\mathcal{O}}\right\rangle$

$$
\begin{aligned}
\leq & (2-2 \eta)|\nabla A|^{2} \cdot h+\frac{4}{2-2 \eta} \frac{\left(h^{\prime}\right)^{2}}{h}|\nabla v|^{2} v^{2}|A|^{2} \\
& +\eta h|\nabla v|^{2}|A|^{2}+\frac{\gamma^{2}}{\eta}\left|\beta_{\varepsilon}^{\prime}\right|^{2}\left|P_{T M} \nu_{\mathcal{O}}\right|^{2} v^{2} \cdot \frac{\left(h^{\prime}\right)^{2}}{h} \cdot|A|^{2} .
\end{aligned}
$$

Combining (8.12)-(8.13) we thus find that

$$
\begin{aligned}
\left(\frac{d}{d t}-\Delta\right) G \leq & -T_{3}\left(v^{2}\right) \cdot e^{\gamma \alpha_{\varepsilon}}|A|^{4}-T_{4}\left(v^{2}\right) \cdot e^{\gamma \alpha_{\varepsilon}}|A|^{2}|\nabla v|^{2}-T_{5}^{(\varepsilon)}\left(v^{2}\right) \cdot G \\
& -\eta e^{\gamma \alpha_{\varepsilon}} h \cdot|\nabla A|^{2}-\frac{1}{h\left(v^{2}\right)}\left\langle\nabla\left(h\left(v^{2}\right)\right), \nabla G\right\rangle+M^{4} \gamma^{-2} G,
\end{aligned}
$$

where

$$
\begin{aligned}
& T_{3}(y):=2 h^{\prime}(y) \cdot y-(2+\eta) h(y), \\
& T_{4}(y):=4 h^{\prime \prime}(y) \cdot y-\left(4+\frac{2}{1-\eta}\right) \cdot \frac{y \cdot\left(h^{\prime}(y)\right)^{2}}{h(y)}+6 h^{\prime}(y)-\eta \cdot h,
\end{aligned}
$$

and

$$
T_{5}^{(\varepsilon)}=\left|\beta_{\varepsilon}^{\prime}\right|\left\langle\nu, \nu_{\mathcal{O}}\right\rangle_{+}+\left|P_{T M} \nu_{\mathcal{O}}\right|^{2}\left(\frac{\gamma}{4} \beta_{\varepsilon}^{\prime \prime}-\gamma^{2}\left(1+\eta^{-1}\right)\left|\beta_{\varepsilon}^{\prime}\right|^{2} \cdot \frac{\left(h^{\prime}\left(v^{2}\right)\right)^{2} v^{2}}{h^{2}\left(v^{2}\right)}\right)
$$

need all be evaluated at $y=v^{2}$, and thus, by assumption, for arguments in the interval $\left[1, M^{2}\right]$.

We will show that all the above terms are strictly positive for $h(y)=y \cdot e^{k y}$ provided $k, \eta$ and $\gamma$ are chosen suitably (depending on the given numbers $M$ and $\ell)$.

We choose $k:=\left(24 M^{2}\right)^{-1}$ and consider the function $h(y)=y \cdot e^{k y}$, whose derivatives are given by

$$
h^{\prime}(y)=h(y) \cdot\left(\frac{1}{y}+k\right), \quad h^{\prime \prime}(y)=h(y) \cdot\left(\frac{2 k}{y}+k^{2}\right) .
$$

Now selecting $\eta$ as $\eta=\frac{k}{2}$ we obtain that the first term in (8.14) is positive, namely

$$
T_{3}(y)=(2 k y-\eta) h(y) \geq \frac{3}{2} k h(y)
$$

for any $y \in\left[1, M^{2}\right]$ which we recall is the range of $v^{2}$ for the points we consider.

Furthermore, as $\frac{2}{1-\eta}=2(1+\lambda \eta)$ for $\lambda=\frac{1}{1-\eta} \leq \frac{48}{47}$, we can bound

$$
\begin{aligned}
T_{4}(y) & =\frac{h(y)}{y} \cdot\left[4\left(2 k+k^{2} y\right) y-(6+2 \lambda \eta)(1+k y)^{2}+6(1+k y)-\eta y\right] \\
& =\frac{h(y)}{y} \cdot\left[2 k y-\eta(2 \lambda+y)+4 k^{2} y^{2}-4 \lambda \eta k y-(6+2 \lambda \eta) k^{2} y^{2}\right] \\
& =\frac{h(y)}{y}\left[2 k y-\frac{k}{2} y-k \lambda+4 k^{2} y^{2}-2 \lambda k^{2} y-(6+\lambda k) k^{2} y^{2}\right] \\
& \geq \frac{h(y)}{y} \cdot\left[\frac{3}{8} k y-6 k^{2} y^{2}\right] \geq \frac{1}{8} k h(y) .
\end{aligned}
$$

We recall that so far we have only imposed an upper bound on $\gamma$, namely $\gamma \in$ $\left(0, \gamma_{0}\right), \gamma_{0}=\gamma_{0}(\eta, \ell)$ the number given by Lemma 8.1. We shall now prove that for $\gamma$ chosen small enough (depending on $\eta$ and $k$ ) also $T_{5}^{(\varepsilon)}$ will be positive. 
Namely, as $\frac{\left(h^{\prime}(y)\right)^{2} \cdot y}{h^{2}(y)} \leq \frac{\left(h^{\prime}(y)\right)^{2} \cdot y}{h(y)}=e^{k y}(1+k y)^{2} \leq 2$ for $y \in\left[1, M^{2}\right]$, we select $\gamma \in\left(0, \gamma_{0}\right)$ small enough to assure that

$$
2 \gamma\left(1+\eta^{-1}\right) \cdot C_{1} \leq \frac{1}{8}
$$

$C_{1}=C_{1}(\ell)$ as in (8.5), in order to get

$$
T_{5}^{\varepsilon} \geq\left|\beta_{\varepsilon}^{\prime}\right|\left\langle\nu, \nu_{\mathcal{O}}\right\rangle_{+}+\frac{\gamma}{8}\left|P_{T M} \nu_{\mathcal{O}}\right|^{2} \beta_{\varepsilon}^{\prime \prime} .
$$

All in all we thus conclude that for points $p$ with $v(p) \leq M$ and $|A(p)| \geq \bar{F}$

$$
\begin{aligned}
\left(\frac{d}{d t}-\Delta\right) G+\frac{1}{h}\langle\nabla h, \nabla G\rangle \leq & -\frac{k}{2} h \cdot e^{\gamma \alpha_{\varepsilon}}|\nabla A|^{2}-\frac{3 k}{2}|A|^{2} \cdot G+\gamma^{-2} M^{4} \cdot G \\
& -\frac{k}{8}|\nabla v|^{2} \cdot G-\left[\frac{\gamma}{8} \beta_{\varepsilon}^{\prime \prime}\left|P_{T M} \nu_{\mathcal{O}}\right|^{2}+\frac{1}{2}\left|\beta_{\varepsilon}^{\prime}\right|\left\langle\nu, \nu_{\mathcal{O}}\right\rangle_{+}\right] \cdot G .
\end{aligned}
$$

This implies the claim of the lemma as we may further increase the number $\bar{F}=$ $\bar{F}_{\gamma}$ determined originally in Lemma 8.1 in order to achieve that $\gamma^{-2} M^{4} \leq \frac{k}{2} \bar{F}^{2}$, allowing us to absorb the third term into the second term on the right-hand side.

We now localise these estimates to be able to apply the maximum principle in halfspaces.

Proposition 8.3. Given any level $\ell \in \mathbb{R}$ and any numbers $M \geq 1$ and $Q<\infty$, there exists a constant $C$ depending only on $\ell, M, Q$ and the obstacle such that for solutions $\left(M_{t}\right)_{t}$ of (3.2) evolving from an initial hypersurface $M_{0}=\operatorname{graph}\left(u_{0}\right)$ disjoint from the obstacle and with $\sup u_{0} \leq Q$ that satisfy

$$
v \leq M \quad \text { on } \quad M_{t} \cap\left\{x^{n+2} \geq \ell-1\right\} \quad \text { for every } t \geq 0,
$$

the second fundamental form is controlled on $M_{t} \cap\left\{x^{n+2} \geq \ell\right\}$ by

(i) (ii)

$$
(U-\ell)^{4} \cdot|A|^{2} \leq \frac{C}{t}
$$

for $t \in(0,1]$ and

$$
(U-\ell)^{4} \cdot|A|^{2} \leq C \cdot\left(1+\sup _{M_{0} \cap\left\{x^{n+2} \geq \ell\right\}}(U-\ell)^{4} \cdot|A|^{2}\right) .
$$

for all $t \geq 0$.

This proposition is an immediate corollary of the subsequent Lemma 8.4 and the maximum principle.

Lemma 8.4. Let $\ell, M, Q \in \mathbb{R}$ and $\left(M_{t}\right)_{t}$ be as in Proposition 8.3. Let $G$ be the second fundamental form quantity considered in Lemma 8.2. Define

$$
w_{0}=(U-\ell)^{4} \cdot G \quad \text { for all } t \geq 0
$$

and

$$
w_{1}=t(U-\ell)^{4} \cdot G+\lambda(U-\ell)^{4} v^{2} \quad \text { for } t \in[0,1] .
$$

Then there exists a constant $D$ such that

$$
\left(\frac{d}{d t}-\Delta\right) w_{i} \leq 0, \quad i=0,1,
$$

in every point where the respective function fulfils $w_{i} \geq D$ and $\nabla w_{i}=0$. 
Proof. We may assume that $\ell=0$. Let $\vartheta \in\{0,1\}$ and set

$$
w:=((1-\vartheta)+t \vartheta) U^{4} G+\vartheta \lambda U^{4} v^{2},
$$

where $\lambda$ is a large constant that will be fixed later. This allows us to consider the two cases simultaneously. If $\vartheta=0$, we obtain a priori estimates up to $t=0$ provided that $|A|^{2}$ is initially bounded. If $\vartheta=1$, we obtain local in time a priori estimates.

We first observe that since $\left\{x^{n+2}=Q\right\}$ lies above $M_{0}$, it must be disjoint from the obstacle and consequently serves as upper barrier for $U$ for all times. In addition to the $C^{1}$-estimates we have furthermore bounds on $\alpha_{\varepsilon}$ thanks to Corollary 6.4. Consequently, if $w$ is large, say $w \geq D$, then also $|A|^{2}$ must be large. In particular, for a suitable choice of $D$, it is enough to consider points with $|A|^{2} \geq \bar{F}$, the constant of Lemma 8.2. We can thus estimate, using Lemmas 5.2 and 8.2

$$
\begin{aligned}
\left(\frac{d}{d t}-\Delta\right) w= & \vartheta U^{4} G+4((1-\vartheta)+t \vartheta) U^{3} G(\dot{U}-\Delta U) \\
& +((1-\vartheta)+t \vartheta) U^{4}(\dot{G}-\Delta G)+4 \vartheta \lambda U^{3} v^{2}(\dot{U}-\Delta U) \\
& +2 \vartheta \lambda U^{4} v(\dot{v}-\Delta v) \\
& -12((1-\vartheta)+t \vartheta) U^{2} G|\nabla U|^{2}-8((1-\vartheta)+t \vartheta) U^{3}\langle\nabla U, \nabla G\rangle \\
& -12 \vartheta \lambda U^{2} v^{2}|\nabla U|^{2}-16 \vartheta \lambda U^{3} v\langle\nabla U, \nabla v\rangle-2 \vartheta \lambda U^{4}|\nabla v|^{2} \\
\leq & \vartheta U^{4} G+4((1-\vartheta)+t \vartheta) U^{3} G \frac{\alpha_{\varepsilon}}{v} \\
& +((1-\vartheta)+t \vartheta) U^{4}\left(-\frac{k}{8} G|A|^{2}-\frac{1}{2}\left|\beta_{\varepsilon}^{\prime}\right|\left\langle\nu, \nu_{\mathcal{O}}\right\rangle+G-\frac{1}{h}\langle\nabla h, \nabla G\rangle\right) \\
& +4 \vartheta \lambda U^{3} v^{2} \frac{\alpha \varepsilon}{v} \\
& +2 \vartheta \lambda U^{4} v\left(\frac{-|A|^{2} v}{(8.15)}-\frac{2}{v}|\nabla v|^{2}+v^{2}\left\langle\nabla \alpha_{\varepsilon}, e_{n+2}\right\rangle\right) \\
& -12((1-\vartheta)+t \vartheta) U^{2} G|\nabla U|^{2}-8((1-\vartheta)+t \vartheta) U^{3}\langle\nabla U, \nabla G\rangle \\
& -12 \vartheta \lambda U^{2} v^{2}|\nabla U|^{2}-16 \vartheta \lambda U^{3} v\langle\nabla U, \nabla v\rangle-2 \vartheta \lambda U^{4}|\nabla v|^{2} .
\end{aligned}
$$

Using that $G \leq C|A|^{2}$, we can use the first underlined term above to absorb (upto an additive constant $C$ ) the second term of the right-hand side. We drop the first term of the penultimate line. Provided $\lambda$ is chosen sufficiently large, we can furthermore absorb the first term on the right hand side into the second underlined term. Estimating also the penultimate term using Young's inequality and bounding the first order terms by a constant, this reduces the above inequality to

$$
\begin{aligned}
\left(\frac{d}{d t}-\Delta\right) w \leq & -c_{1}((1-\vartheta)+t \vartheta) U^{4} G^{2}-\vartheta \lambda U^{4} v^{2}|A|^{2}-\left(6-\frac{1}{4}\right) \vartheta \lambda U^{4}|\nabla v|^{2}+C \\
& +I+I I+I I I_{\varepsilon}
\end{aligned}
$$

for some $c_{1}>0$ and a constant $C<\infty$ which may also depend on $\lambda$.

Here $I$ and $I I$ stand for the terms appearing on the right hand side of (8.15) that contain $\nabla G$ while

$$
I I I_{\varepsilon}:=2 \vartheta \lambda U^{4} v^{3}\left\langle\nabla \alpha_{\varepsilon}, e_{n+2}\right\rangle-((1-\vartheta)+t \vartheta) U^{4} G \frac{1}{2}\left|\beta_{\varepsilon}^{\prime}\right|\left\langle\nu, \nu_{\mathcal{O}}\right\rangle_{+} .
$$

Since we only consider points at which $\nabla w=0$ we can replace $\nabla G$ in both $I$ and $I I$ using

$$
\begin{aligned}
0 & =\nabla w \\
& =4((1-\vartheta)+t \vartheta) U^{3} G \nabla U+((1-\vartheta)+t \vartheta) U^{4} \nabla G+4 \vartheta \lambda U^{3} v^{2} \nabla U+2 \vartheta \lambda U^{4} v \nabla v
\end{aligned}
$$


Recall furthermore that

$$
|\nabla v|^{2}=g^{i j} \nabla_{i} v \nabla_{j} v=v^{4} g^{i j} A_{i}^{k} \nabla_{k} U A_{j}^{l} \nabla_{l} U \leq v^{4}|\nabla U|^{2}|A|^{2} \leq v^{4}|A|^{2} \leq c G,
$$

compare (5.5) and that $h(y)=y e^{k y}$ so, writing for short $\nabla h$ for $\nabla\left(h\left(v^{2}\right)\right)$,

$$
\frac{\nabla h}{h}=\frac{h^{\prime}}{h} \nabla\left(v^{2}\right)=\left(\frac{1}{v^{2}}+k\right) 2 v \nabla v
$$

which, thanks to the $C^{1}$-estimates is bounded by $C|\nabla v| \leq C|A| \leq C G^{1 / 2}$. We can thus estimate

$$
\begin{aligned}
I= & -((1-\vartheta)+t \vartheta) U^{4} \frac{1}{h}\langle\nabla h, \nabla G\rangle \\
\leq & C((1-\vartheta)+t \vartheta) U^{3} v G|\langle\nabla v, \nabla U\rangle|+C \vartheta \lambda v^{3} U^{3}|\langle\nabla v, \nabla U\rangle| \\
& \quad+4 \vartheta \lambda U^{4}\left(1+k v^{2}\right)|\nabla v|^{2} \\
\leq & C((1-\vartheta)+t \vartheta) U^{3} G^{3 / 2}+C \vartheta \lambda U^{3}|A|+5 \vartheta \lambda U^{4}|\nabla v|^{2},
\end{aligned}
$$

where we used that $k v^{2} \leq \frac{1}{24}$ as well as that $v$ is bounded in the last step. Using Young's inequality, we can absorb the first two terms of this estimate into the first two terms of the right hand side of (8.16) and another additive constant $C(\lambda)$, while the last term is absorbed into the third term of (8.16).

Furthermore, the terms appearing in

$$
\begin{aligned}
I I & =-8((1-\vartheta)+t \vartheta) U^{3}\langle\nabla U, \nabla G\rangle \\
& =32((1-\vartheta)+t \vartheta) U^{2} G|\nabla U|^{2}+32 \vartheta \lambda U^{2} v^{2}|\nabla U|^{2}+16 \vartheta \lambda U^{3} v\langle\nabla U, \nabla v\rangle \\
& \leq C((1-\vartheta)+t \vartheta) U^{2} G+C+C \vartheta \lambda U^{3}|A|
\end{aligned}
$$

can also be absorbed into the first two terms on the right hand side of (8.16) and a constant.

Finally, to analyse $I I I_{\varepsilon}$, we recall that $\left\langle\nabla \alpha_{\varepsilon}, e_{n+2}\right\rangle=\beta_{\varepsilon}^{\prime}\left(\left\langle\nu_{\mathcal{O}}, e_{n+2}\right\rangle-\frac{1}{v}\left\langle\nu_{\mathcal{O}}, \nu\right\rangle\right) \leq$ $\frac{\left|\beta_{\varepsilon}^{\prime}\right|}{v}\left\langle\nu_{\mathcal{O}}, \nu\right\rangle$. Thus

$$
\begin{aligned}
I I I_{\varepsilon} & \leq-\frac{1}{2}\left\langle\nu, \nu_{\mathcal{O}}\right\rangle_{+}\left|\beta_{\varepsilon}^{\prime}\right| \cdot\left[\left((1-\vartheta+t \vartheta) U^{4} G-4 \vartheta \lambda U^{4} v^{2}\right]\right. \\
& =-\frac{1}{2}\left\langle\nu, \nu_{\mathcal{O}}\right\rangle_{+}\left|\beta_{\varepsilon}^{\prime}\right| \cdot\left[w-5 \vartheta \lambda U^{4} v^{2}\right]
\end{aligned}
$$

is negative in points where $w \geq D$ provided $D$ is chosen sufficiently large.

All in all we thus conclude that we can fix a number $\lambda \geq 1$ so that the estimate

$$
\left(\frac{d}{d t}-\Delta\right) w \leq-\frac{c_{1}}{4}((1-\vartheta)+t \vartheta) U^{4} G^{2}+C
$$

holds in every point in which $\nabla w=0$ and $w \geq D$.

We finally remark that in points where $w$ is large the first term in this estimate dominates since also

$$
((1-\vartheta)+t \vartheta) U^{4} G^{2} \geq((1-\vartheta)+t \vartheta)^{2} U^{4} G^{2}=U^{-4}\left(w-\vartheta \lambda U^{4} v^{2}\right)^{2}
$$

must be large as $U$ and $v$ are bounded above and as we only consider times $t \in[0,1]$ in case $\vartheta=1$. Thus increasing $D$ further allows us to absorb the second term and yields the claim.

8.1. $C^{k}$-estimates for solutions of the approximate problem. In order to guarantee the existence of solutions to the penalised flow (3.2) for all time, we show that, for each fixed number $\varepsilon>0$, solutions of (3.2) satisfy $C^{k}$-estimates for all positive times.

We stress that these estimates are not uniform in $\varepsilon$ and indeed that no such uniform control is possible as already the solutions of the stationary graphical obstacle problem are in general only in $C^{1,1}$, see [8]. As such we shall refrain 
from writing down the explicit form of most terms and for the most part use the notation $B * C$ to denote arbitrary linear combinations of traces of $B \otimes C$ with respect to the metric.

Recall that under the flow (3.2), the metric evolves according to (5.2), so that its Christoffel-symbols $\Gamma$ satisfy

$$
\frac{d}{d t} \Gamma=\nabla A * A+\nabla \alpha_{\varepsilon} * A+\alpha_{\varepsilon} * \nabla A
$$

Since $\frac{d}{d t} \nabla B=\nabla \frac{d}{d t} B+\frac{d}{d t} \Gamma * B$ for any tensor $B$, we thus get

$$
\frac{d}{d t} \nabla^{m} A=\nabla^{m} \frac{d}{d t} A+\nabla^{a_{1}} A * \nabla^{a_{2}} A * \nabla^{a_{3}} A+\nabla^{a_{1}} \alpha_{\varepsilon} * \nabla^{a_{2}} A * \nabla^{a_{3}} A,
$$

where $a_{i} \in \mathbb{N}_{0}$ range over all triples with $a_{1}+a_{2}+a_{3}=m$.

The evolution equation for the second fundamental form for the general flow (5.1) is known to be

$$
\frac{d}{d t} A_{i j}=\nabla_{i} \nabla_{j} f-f A_{i}^{k} A_{k j}
$$

which implies

$$
\frac{d}{d t} A_{i j}-\Delta A_{i j}=|A|^{2} A_{i j}-2 H A_{i}^{k} A_{k j}+(H-f) A_{i}^{k} A_{k j}-\nabla_{i} \nabla_{j}(H-f) .
$$

For our flow we thus have

$$
\left(\frac{d}{d t}-\Delta\right) A=-\nabla^{2} \alpha_{\varepsilon}+\alpha_{\varepsilon} * A * A+A * A * A .
$$

Using that $\Delta \nabla^{m} A=\nabla^{m} \Delta A+\nabla^{a_{1}} A * \nabla^{a_{2}} A * \nabla^{a_{3}} A$, compare (4.1), we get

$$
\begin{aligned}
\left(\frac{d}{d t}-\Delta\right)\left|\nabla^{m} A\right|^{2}= & -2\left|\nabla^{m+1} A\right|^{2}+\nabla^{m+2} \alpha_{\varepsilon} * \nabla^{m} A \\
& +\left(\nabla^{a_{1}} A+\nabla^{a_{1}} \alpha_{\varepsilon}\right) * \nabla^{a_{2}} A * \nabla^{a_{3}} A * \nabla^{m} A,
\end{aligned}
$$

$a_{1}+a_{2}+a_{3}=m$. Since $A$ is bounded on $M_{t} \cap\left\{x^{n+2} \geq \ell\right\}$ and since in such regions the depth of penetration is controlled by the results of Section 6 , we conclude that in this region

$$
\left(\frac{d}{d t}-\Delta\right)\left|\nabla^{m} A\right|^{2} \leq-2\left|\nabla^{m+1} A\right|^{2}+C \cdot\left|\nabla^{m} A\right|^{2}+C+C \varepsilon^{-2(m+2)},
$$

with $C$ depending on $\ell$, the $C^{m+3}$-norm of the obstacle $\psi$, bounds on $A, \nabla A, \ldots$, $\nabla^{m-1} A$, and either a lower bound on $t$ or a bound on the second fundamental form of the initial hypersurface.

Remark 8.5. For fixed $\varepsilon>0$, we deduce iteratively estimates for $\left|\nabla^{m} A\right|, m=$ $1,2, \ldots$ for solutions of (3.2) of the following form:

(i) for any $\ell \in \mathbb{R}$, any $0<\tau$ and any $m \in \mathbb{N}$, there is a constant $C$ depending on $\varepsilon>0$, on the obstacle and on local $C^{1}$-bounds of $M_{0} \cap\left\{x^{n+2}>\ell-1\right\}$, so that $\left|\nabla^{m} A\right| \leq C$ in $M_{t} \cap\left\{x^{n+2} \geq \ell\right\}, t \geq \tau$.

(ii) if $M_{0} \cap\left\{x^{n+2}>\ell-1\right\}$ is additionally in $C^{m+2}$, then these estimates are valid up to time $t=0$, i. e. $\left|\nabla^{m} A\right| \leq C$ in $M_{t} \cap\left\{x^{n+2} \geq \ell\right\}, t \in[0, \infty)$.

Proof. We may proceed as in the proof in the situation without obstacles, see [19, Theorem 5.9], after replacing the set where $u<0$ with the one where $U>0$ due to the different orientation of the graphs. If the derivatives $\left|\nabla^{k} A\right|, 1 \leq k \leq m-1$, are already uniformly bounded in the set considered, the evolution equations for $\left|\nabla^{m} A\right|^{2}$ and $\left|\nabla^{m-1} A\right|^{2}$ are of the same form as in the proof of [19, Theorem 5.9]. Note that the constants $c$ will now depend on $\frac{1}{\varepsilon}$. This, however, does not cause problems as we do not claim that these estimates are independent of $\varepsilon$. When we compute the evolution equation of

$$
t U^{2}\left|\nabla^{m} A\right|^{2}+\lambda\left|\nabla^{m-1} A\right|^{2},
$$

we get an additional term $2 t u \alpha_{\varepsilon}\left\langle\nu, e_{n+2}\right\rangle\left|\nabla^{m} A\right|^{2}$, which can easily be absorbed. The rest of the argument carries over to the present situation. 


\section{Existence OF APPROXimate SOLUtions}

We construct smooth approximate solutions to (2.1) depending on parameters

- $\varepsilon \in(0,1)$ controlling the penalisation,

- $L \in \mathbb{R}$, the height at which we truncate our initial value,

- $R>3$, the radius of the ball on which we solve a Dirichlet problem, and

- $\delta \in(0,1)$ to mollify both the truncated initial values and the obstacle.

Given an obstacle $\mathcal{O}$ with $\partial \mathcal{O}=\operatorname{graph}(\psi)$ for a $C_{\text {loc }}^{1,1}$-function $\psi$ as described in Definition 2.1 , we extend $\psi$ by $-\infty$ to $\mathbb{R}^{n+1}$. Then we mollify $\mathcal{O}$ and consider the obstacles $\mathcal{O}^{\delta}, \delta \in(0,1]$, characterised by $\partial \mathcal{O}^{\delta}=\operatorname{graph}\left(\psi_{\delta}\right)$, where

$$
\psi_{\delta}=\psi * \eta_{\delta}
$$

for a smooth mollification kernel $\eta_{\delta}=\delta^{-(n+1)} \eta(\cdot / \delta)$, supp $\eta \subset B_{1}(0)$, and let

$$
\alpha_{\varepsilon}^{\delta}=\beta_{\varepsilon} \circ \operatorname{dist}_{\partial \mathcal{O}^{\delta}}
$$

be the corresponding penalisation function.

We remark that all results derived in the previous sections (except for the higher order estimates of Remark 8.5) are valid with constants independent of $\delta$ for this whole family of obstacles as $\left(\mathcal{O}^{\delta}\right)_{\delta \in(0,1]}$ satisfy uniform $C_{\mathrm{loc}}^{2}$-estimates.

We remark that mollifying the initial value $u_{0}$ with the same kernel ensures that $u_{0} \geq \psi$ remains true after mollification.

In order to apply the results derived in the previous sections, we shall furthermore only consider parameters so that

$$
\varepsilon \leq \varepsilon_{0}(L) \text { the constant of Lemma } 6.1
$$

and so that $R$ is large enough to guarantee that the initial map $u_{0}$ satisfies

$$
u_{0} \leq L-1 \text { outside } B_{R / 2}(0) .
$$

We then have the following existence result for approximate solutions.

Proposition 9.1. Let $u_{0}$ and $\mathcal{O}$ with $\partial \mathcal{O}=\left.\operatorname{graph} \psi\right|_{\mathcal{P}}$ be an initial map and an obstacle as described in Definition 2.1 and let $\mathcal{O}^{\delta}, \delta \in(0,1]$, be the mollified obstacles as described above.

Then for every quadruple $(\varepsilon, \delta, L, R) \in(0,1)^{2} \times \mathbb{R} \times[3, \infty)$ of parameters for which the assumptions (9.1) and (9.2) are satisfied, there exists a smooth solution $u_{\varepsilon, R}^{\delta, L}: B_{R}(0) \times[0, \infty) \rightarrow \mathbb{R}$ to

$$
\begin{cases}\dot{u}=\sqrt{1+|D u|^{2}} \cdot\left(\operatorname{div}\left(\frac{D u}{\sqrt{1+|D u|^{2}}}\right)+\alpha_{\varepsilon}^{\delta}\right) & \text { in } B_{R}(0) \times[0, \infty), \\ u=L & \text { on } \partial B_{R}(0) \times[0, \infty), \\ u(\cdot, 0)=\left(\max \left\{u_{0}, L\right\}\right)_{\delta}=\max \left\{u_{0}, L\right\} * \eta_{\delta} & \text { in } B_{R}(0) .\end{cases}
$$

Furthermore, for any $\ell>L+2$, there exists a constant $C=C\left(u_{0}, \mathcal{O}, \ell\right)$ such that

$$
\left|D u_{\varepsilon, R}^{\delta, L}(x, t)\right|+\sqrt{t} \cdot\left|D^{2} u_{\varepsilon, R}^{\delta, L}(x, t)\right|+\sqrt{t} \cdot\left|\frac{d}{d t} u_{\varepsilon, R}^{\delta, L}(x, t)\right| \leq C
$$

in every $(x, t) \in B_{R} \times[0, \infty)$ with $u_{\varepsilon, R}^{\delta, L}(x, t) \geq \ell$.

Here, the function $\alpha_{\varepsilon}^{\delta}$ is evaluated at the point $\left(x, u_{\varepsilon, R}^{\delta, L}(x, t)\right)$ on the evolving hypersurface graph $u_{\varepsilon, R}^{\delta, L}(\cdot, t)$.

Proof. The choice of $R$ implies that the smooth initial map $\left(\max \left\{u_{0}, L\right\}\right)_{\delta}$ is constant near $\partial B_{R}(0)$, so that compatibility conditions of any order are fulfilled for the initial value problem (9.3). As (9.3) is a quasilinear parabolic partial differential equation on a compact domain, we may apply standard parabolic theory as found 
e. g. in $[7,14]$ and as carried out in detail for a similar problem in $[9$, Ch. 2] to obtain the existence of a smooth solution $u \equiv u_{\varepsilon, R}^{\delta, L}$ defined on a maximal time interval $[0, T), T>0$, where $T<\infty$ only if sup $|D u|$ becomes unbounded in finite time.

To establish long time existence it is thus sufficient to show that the derivatives of $u$ remain bounded for all times which we shall prove using a combination of standard techniques for mean curvature flow as well as the evolution equations derived in the previous sections. We remark that in this part of the proof we do not claim that any of the derived bounds are independent of the choice of the parameters but will rather prove the uniform a priori bounds (9.4) separately later on.

Let $\varepsilon, \delta, R, L$ be any fixed parameters as in the proposition. To begin with, we observe that since $\alpha_{\varepsilon}^{\delta} \geq 0$, any constant function is a subsolution of the equation; in particular the constant $L$ serves as a lower barrier for $u$. Furthermore, the constant $\max \left\{\sup \psi, \sup u_{0}, L\right\}$ is a solution to the flow equation as $\alpha_{\varepsilon}^{\delta}$ vanishes on its graph, so it is an upper barrier and our solution remains uniformly bounded for all times.

We remark that due to our choice of $R$, we have $u(x, t) \geq L>\psi(x)$ for any $|x| \geq R / 2$ and any $t>0$ so $u$ evolves according to graphical mean curvature flow in any annulus $\left(B_{\rho} \backslash B_{\sigma}(0)\right) \times[0, T)$ with $R / 2<\sigma<\rho<R$. Hence the interior estimates for solutions of graphical mean curvature flow of K. Ecker and G. Huisken [5], in particular [5, Theorem 3.1 and Theorem 3.4], apply in this region and yield that all derivatives of $u=u_{\varepsilon, R}^{\delta, L}$ are bounded on $[\tau, T)$, for any $0<\tau<T, T$ the maximal existence time, so $D u$ cannot blow-up in finite time in this region.

The arguments of the proof of [12, Theorem 2.1], which compare the evolving hypersurface with barriers previously used in the study of the Dirichlet problem for minimal hypersurfaces, furthermore yield uniform bounds on $|D u|$ on $\partial B_{R} \times[0, T)$. As we have already obtained uniform gradient bounds on $\partial B_{\frac{3 R}{4}} \times[\tau, T)$, we hence obtain uniform gradient bounds on $\left(B_{R} \backslash B_{\frac{3 R}{4}}\right) \times[\tau, T)$ by applying the maximum principle to the evolution equation of the gradient function $v$ on this domain. Finally, the uniform parabolicity of the equation and the fact that compatibility conditions of all orders are satisfied, allow us to apply standard parabolic theory as found e.g. in [7, 14], see in particular [14, Theorem IV.5.2], to conclude that derivatives of $u$ of arbitrary order are bounded on $\left(B_{R} \backslash B_{\frac{3 R}{4}}\right) \times[\tau, T)$.

To derive estimates in the interior, say on $B_{\frac{3 R}{4}}$, we can now apply the maximum principle on $B_{\frac{3 R}{4}}$ to the various evolution equations derived in the previous sections since we have already obtained bounds on the annulus and thus in particular on $\partial B_{\frac{3 R}{4}}$; namely gradient estimates now follow from Lemma 5.2 and Remark 5.3, estimates on the second fundamental form follow from Lemma 8.2 and higher order estimates follow from (8.17). Having thus proven that all derivatives of $u$ remain bounded up to the maximal existence time $T$, we hence conclude that $T=\infty$ as otherwise $u(\cdot, t)$ would converge to a smooth limit $u(\cdot, T)$ from which we could restart the flow and hence obtain a solution defined on a larger interval, contradicting the maximality of $T$.

Having thus established long time existence of each approximate solution, we finally need to prove that these approximate solutions satisfy the estimate (9.4) for a constant $C$ that is independent of the approximation parameters $\delta, L, \varepsilon, R$. As the initial hypersurfaces $\left(M_{\varepsilon, R}^{\delta, L}\right)_{t=0}$ satisfy uniform gradient bounds in $\left\{x^{n+2} \geq \ell-2\right\}$, Proposition 7.1 yields uniform bounds on $\left|D u_{\varepsilon . R}^{\delta, L}\right|$ in points with $u_{\varepsilon, R}^{\delta, L} \geq \ell-1$ as required. These uniform gradient bounds finally allow us to apply Proposition 8.3 
to obtain the desired uniform bound on the second fundamental form and thus on the second term in (9.4) in points where $u_{\varepsilon, R}^{\delta, L} \geq \ell$.

These estimates then imply the claim on the time derivative made in (9.4) since $u$ solves equation (9.3) and since the penetration depth, and thus $\alpha_{\varepsilon}^{\delta}$, is a priori controlled according to Corollary 6.4 .

\section{Proofs OF the MAIN RESUlts}

We are now able to prove the existence of viscosity solutions of graphical mean curvature flow with obstacles as claimed in Theorems 3.1 and 3.3.

Proof of Theorem 3.1. Let $\mathcal{O}$ and $u_{0}$ be an obstacle and initial condition as in Definition 2.1 and let $u^{i}=u_{\varepsilon_{i}, R_{i}}^{\delta_{i}, L_{i}}$ be any sequence of approximate solutions as constructed in Proposition 9.1 for which $\left(\varepsilon_{i}, L_{i}, \delta_{i}, R_{i}\right) \rightarrow(0,-\infty, 0, \infty)$.

Then the uniform $C^{2 ; 1}$ estimates stated in (9.4) allow us to apply the variant of the theorem of Arzelà-Ascoli from [19, Lemma 7.3]: we obtain a subsequence $u^{i}$ converging to a limiting function $\tilde{u}: \mathbb{R}^{n+1} \times[0, \infty) \rightarrow \mathbb{R} \cup\{-\infty\}$ which induces a pair $(\Omega, u)$ consisting of

$$
\Omega:=\{(x, t): u(x, t)>-\infty\} \subset \mathbb{R}^{n+1} \times[0, \infty)
$$

and the restriction $u:=\left.\tilde{u}\right|_{\Omega}: \Omega \rightarrow \mathbb{R}$. Here the convergence of $u^{i} \rightarrow \tilde{u}$ is pointwise everywhere and in $C_{l o c}^{1, \alpha ; 0, \alpha}(\Omega \cap\{t>0\})$ for every $\alpha \in(0,1)$.

We recall furthermore, that the graphical velocity of the approximate solutions is controlled by (9.4). Therefore the approximate solutions satisfy uniform parabolic Hölder estimates up to time $t=0$ on any compact subsets of $\Omega$ and so the obtained limit $u$ is in $C_{l o c}^{0}(\Omega)$ and attains the desired initial value $u(0)=u_{0}$.

We now prove that $u$ is a viscosity solution of

$$
\min \left\{\dot{u}-\sqrt{1+|D u|^{2}} \cdot \operatorname{div}\left(\frac{D u}{\sqrt{1+|D u|^{2}}}\right), u-\psi\right\}=0 .
$$

We recall that $u$ is a viscosity subsolution for the above operator if for any point $\left(x_{0}, t_{0}\right)$, the left-hand side of (10.1) is nonpositive for all $C^{2}$-functions $\varphi$ satisfying $\varphi\left(x_{0}, t_{0}\right)=u\left(x_{0}, t_{0}\right)$ as well as $u(x, t) \leq \varphi(x, t)$ for all $(x, t) \in \Omega$ with $t<t_{0}$.

To begin with, we observe that the estimates on the penetration depth derived in Section 6 imply that

$$
u(x, t) \geq \psi(x)
$$

for every $(x, t) \in \Omega$. We can thus distinguish between points with $u\left(x_{0}, t_{0}\right)>\psi\left(x_{0}\right)$ and points where the hypersurface touches the closure of the obstacle.

In the former case it is clearly enough to show that $u$ is locally a viscosity solution of the graphical mean curvature flow equation

$$
\dot{u}+\sqrt{1+|D u|^{2}} \cdot H=0 .
$$

Given such a point $\left(x_{0}, t_{0}\right)$ in which $u\left(x_{0}, t_{0}\right)>\psi\left(x_{0}\right)$, we observe that in a space time neighbourhood also $u^{i}(x, t) \geq \psi(x)$ for $i$ sufficiently large, since these functions converge locally uniformly to $u$. Consequently the functions $u^{i}$ are classical solutions of (10.2) in this neighbourhood. As we have locally uniform gradient estimates for the functions $u_{i}$, equation (10.2) is uniformly parabolic, so arguing as in [2, Proposition 2.9], we obtain that the limit $u$ is indeed a viscosity solution to $(10.2)$

It remains to consider points $\left(x_{0}, t_{0}\right)$ with $u\left(x_{0}, t_{0}\right)=\psi\left(x_{0}\right)$. First of all, since the second argument in the minimum in (10.1) is zero for every $C^{2}$ function $\varphi$ with $\varphi\left(x_{0}, t_{0}\right)=u\left(x_{0}, t_{0}\right)$, the condition that this minimum is non-positive in the 
viscosity sense is clearly satisfied. It remains to show that $\dot{u}+\sqrt{1+|D u|^{2}} \cdot H \geq 0$ holds in the viscosity sense. But $\alpha_{\varepsilon} \geq 0$, so the functions $u^{i}$ satisfy this inequality classically on the whole domain of definition so that passing to the limit as explained above implies that $u$ itself satisfies the inequality in the viscosity sense. We conclude that $u$ is a viscosity solution to (10.1).

The claimed estimate (3.1) follows from (9.4). For a $C_{\mathrm{loc}}^{1,1}$-initial hypersurface we can furthermore derive bounds on the second fundamental form up to $t=0$ from Lemma 8.4.

Consider finally a point $(x, t) \in \Omega$ with $t>0$ that is not contained in the contact set $\Gamma$, i. e. such that $u(x, t)>\psi(x)$. By uniform convergence we also have $u^{i}>\psi$ in a neighbourhood of $(x, t)$ for sufficiently large $i$. Thus $u^{i}$ evolves by graphical mean curvature flow in this neighbourhood. As the $u^{i}$ satisfy locally uniform gradient estimates we may apply the interior estimates of [5, Theorems $3.1,3.4]$ and deduce smoothness of $u$ in a smaller neighbourhood of $(x, t)$.

Proof of Theorem 3.3. We proceed as in the proof of Theorem 3.1 and consider approximate solutions $u_{\varepsilon, R}^{\delta}$ as in Proposition 9.1 but now with the initial and boundary values in (9.3) replaced with $u(x, t)=u_{0}(x) \equiv \lim _{|y| \rightarrow \infty} u_{0}(y)$ on $\partial B_{R}(0) \times[0, \infty)$ for large $R>0$ and $u(\cdot, 0)=u_{0} * \eta_{\delta}$ in $B_{R}(0)$. Using large spheres near infinity as barriers, we can separate the evolving graph from the obstacle near infinity. Thus $u_{\varepsilon, R}^{\delta}$ solves graphical mean curvature flow without additional terms due to the obstacle outside of a compact set that does not depend on $R$ but may grow in time. In this region, we can thus apply the a priori estimates of [5] and obtain uniform bounds on arbitrary derivatives of $u_{\varepsilon, R}^{\delta}$.

As the additional term $\alpha_{\varepsilon}^{\delta}$ is nonnegative, a hyperplane at height inf $u_{0}-1$ acts as a lower barrier. Therefore $u_{0}$ can at most penetrate into a bounded subset of the obstacle and we can apply the maximum principle with $f_{0}$ equal to a constant in Lemma 6.2. Then we obtain bounds on derivatives of $u_{\varepsilon, R}^{\delta}$ by applying the maximum principle directly (i.e. without localising with $U-\ell$ ) to the evolution equations for $v$ of Lemma 5.2, for $G$ of Lemma 8.2 and to (8.17) for higher order derivatives. This is possible since far away from the origin those quantities are controlled by the estimates of [5], so that we can apply the maximum principle on compact sets. This implies spatial $C^{2}$-estimates that depend neither on $\varepsilon, \delta$ nor $R$ and higher order estimates that depend only on $\varepsilon$ but not on $\delta$ or $R$.

Then arguing as in the proof of Proposition 9.1 yields the analogue of this proposition, in particular estimate $(9.4)$ on all of $B_{R}(0) \times[0, \infty)$. Thus the arguments of the proof of Theorem 3.1 also apply to the present situation and yield the desired result.

\section{Geometric interpretation: BACK to the ORIGinAl PROBlem}

We finally discuss how the graphical solutions constructed in the previous sections can lead to a notion of weak solutions for the original problem of flowing a general (in particular not necessarily graphical) hypersurface $N_{0}$ in $\mathbb{R}^{n+1}$ in the presence of an obstacle $\mathcal{P} \subset \mathbb{R}^{n+1}$. We consider the case of a one-sided obstacle, intuitively speaking an obstacle such that either all or none of its components are enclosed by the initial hypersurface. This includes of course the special case of a connected obstacle.

To be more precise, let $d_{N_{0}}$ be a continuous distance function to $N_{0}$ which has non-vanishing gradient on $N_{0}$ (and thus changes sign as we pass through $N_{0}$ ). We then ask that $d_{N_{0}}$ has constant sign on all of $\mathcal{P}$, say $d_{N_{0}} \mid \mathcal{P}<0$ and consider a 
complete graphical initial hypersurfaces over $\Omega_{0}:=\left\{x: d_{N_{0}}(x)<0\right\}$ and a complete graph over the obstacle as in Definition 2.1. This construction requires no regularity of the initial hypersurface $N_{0}$ or the obstacle $\mathcal{P}$.

Let now $(\Omega, u)$ be the corresponding singularity resolving solution whose existence for all times we have proven above.

Let $\Omega_{t}$ be the time-slice of $\Omega$ at time $t$ as in Definition 2.2 (i). Then $M_{t}:=$ $\operatorname{graph}\left(u(\cdot, t): \Omega_{t} \rightarrow \mathbb{R}\right)$ is a complete hypersurface and $\left(M_{t}\right)_{t \geq 0}$ solves graphical mean curvature flow respecting the obstacle. We can now view $\left(\Omega_{t}\right)_{t}$ as a global weak solution of mean curvature flow respecting the original obstacle: Since $u(\cdot, t) \geq$ $\psi$ for every $t$, the projected flow respects the obstacle in the desired way that $\Omega_{t}$ contains $\mathcal{P}$ for all times and $\partial \Omega_{t}$, which represents a weak analogue of an evolving hypersurface $N_{t}$ in $\mathbb{R}^{n+1}$, remains disjoint from the open obstacle $\mathcal{P}$. Away from the obstacle, [16, Theorem 3.5] ensures that $N_{t}=\partial \Omega_{t}$ represents a weak solution of mean curvature flow in the following sense: For any $t_{0} \in[0, \infty)$, any $t_{1}>t_{0}$ and any smooth closed solution $\left(C_{t}\right)_{\left[t_{0}, t_{1}\right]}$ of mean curvature flow, which is disjoint from the obstacle $\overline{\mathcal{P}}$ on $\left[t_{0}, t_{1}\right]$ and initially disjoint from the evolving hypersurface, i. e. $C_{t_{0}} \cap N_{t_{0}}=\emptyset$, we have that $C_{t}$ remains disjoint from $M_{t}$ on all of $\left[t_{0}, t_{1}\right]$. This represents the analogue of the characterisation of level set flow through the avoidance principle as in [13].

We furthermore remark that having constructed graphical solutions provides the basis for an alternative approach to level-set flow, following the seminal work of L. C. Evans and J. Spruck [6] and it would be of interest to understand if this approach is equivalent to the viscosity approach developed by G. Mercier in [17].

\section{REFERENCES}

1. Luís. Almeida, Antonin Chambolle, and Matteo Novaga, Mean curvature flow with obstacles, Ann. Inst. H. Poincaré Anal. Non Linéaire 29 (2012), no. 5, 667-681.

2. Luis A. Caffarelli and Xavier Cabré, Fully nonlinear elliptic equations, American Mathematical Society Colloquium Publications, vol. 43, American Mathematical Society, Providence, RI, 1995.

3. Michael G. Crandall, Hitoshi Ishii, and Pierre-Louis Lions, User's guide to viscosity solutions of second order partial differential equations, Bull. Amer. Math. Soc. (N.S.) 27 (1992), no. 1, $1-67$.

4. Klaus Ecker, Regularity theory for mean curvature flow, Progress in Nonlinear Differential Equations and their Applications, 57, Birkhäuser Boston Inc., Boston, MA, 2004.

5. Klaus Ecker and Gerhard Huisken, Interior estimates for hypersurfaces moving by mean curvature, Invent. Math. 105 (1991), no. 3, 547-569.

6. Lawrence C. Evans and Joel Spruck, Motion of level sets by mean curvature. I, J. Differential Geom. 33 (1991), no. 3, 635-681.

7. Avner Friedman, Partial differential equations of parabolic type, Prentice-Hall Inc., Englewood Cliffs, N.J., 1964.

8. Claus Gerhardt, Global $C^{1,1}$-regularity for solutions of quasilinear variational inequalities, Arch. Rational Mech. Anal. 89 (1985), no. 1, 83-92.

9. Claus Gerhardt, Curvature problems, Series in Geometry and Topology, vol. 39, International Press, Somerville, MA, 2006.

10. David Gilbarg and Neil S. Trudinger, Elliptic partial differential equations of second order, second ed., Grundlehren der Mathematischen Wissenschaften, vol. 224, Springer-Verlag, Berlin, 1983.

11. Gerhard Huisken and Alexander Polden, Geometric evolution equations for hypersurfaces, Calculus of variations and geometric evolution problems (Cetraro, 1996), Lecture Notes in Math., vol. 1713, Springer, Berlin, 1999, pp. 45-84.

12. Gerhard Huisken, Nonparametric mean curvature evolution with boundary conditions, J. Differential Equations 77 (1989), no. 2, 369-378.

13. Tom Ilmanen, The level-set flow on a manifold, Differential geometry: partial differential equations on manifolds (Los Angeles, CA, 1990), Proc. Sympos. Pure Math., vol. 54, Amer. Math. Soc., Providence, RI, 1993, pp. 193-204. MR 1216585 
14. Olga A. Ladyženskaja, Vsevolod A. Solonnikov, and Nina N. Ural'ceva, Linear and quasilinear equations of parabolic type, Translated from the Russian by S. Smith. Translations of Mathematical Monographs, Vol. 23, American Mathematical Society, Providence, R.I., 1967.

15. Philippe Logaritsch, An obstacle problem for mean curvature flow, Ph.D. thesis, University of Leipzig, 2016.

16. Wolfgang Maurer, Shadows of graphical mean curvature flow, 2016, arXiv:1604.05238 [math.DG], Comm. Anal. Geom., accepted.

17. Gwenael Mercier, Mean curvature flow with obstacles: a viscosity approach, arXiv:1409.7657v1 [math.AP].

18. Gwenaël Mercier and Matteo Novaga, Mean curvature flow with obstacles: existence, uniqueness and regularity of solutions, Interfaces Free Bound. 17 (2015), no. 3, 399-426.

19. Mariel Sáez Trumper and Oliver C. Schnürer, Mean curvature flow without singularities, J. Differential Geom. 97 (2014), no. 3, 545-570.

20. Emanuele Spadaro, Mean-convex sets and minimal barriers, arXiv:1112.4288v1 [math.DG].

Melanie Rupflin, Mathematical Institute, University of Oxford, Radcliffe ObserVAtory Quarter, Woodstock Rd, Oxford OX2 6GG, United Kingdom

Email address: rupflin@maths.ox.ac.uk

Oliver C. Schnürer, Fachbereich Mathematik und Statistik, Universität Konstanz, 78457 Konstanz, Germany

Email address: 0liver.Schnuerer@uni-konstanz.de 